\title{
POD A-POSTERIORI ERROR ESTIMATES FOR LINEAR-QUADRATIC OPTIMAL CONTROL PROBLEMS
}

\author{
F. TRÖLTZSCH AND S. VOLKWEIN
}

\begin{abstract}
The main focus of this paper is on an a-posteriori analysis for the method of proper orthogonal decomposition (POD) applied to optimal control problems governed by parabolic and elliptic PDEs. Based on a perturbation method it is deduced how far the suboptimal control, computed on the basis of the POD model, is from the (unknown) exact one. Numerical examples illustrate the realization of the proposed approach for linear-quadratic problems governed by parabolic and elliptic partial differential equations.
\end{abstract}

\section{Introduction}

Optimal control problems for partial differential equation are often hard to tackle numerically because their discretization leads to very large scale optimization problems. Therefore, different techniques of model reduction were developed to approximate these problems by smaller ones that are tractable with less effort. Among them, the method of proper orthogonal decomposition (POD) and the balanced truncation method seem to be most widely used.

Recently, both approaches have received increasing attention; we refer, e.g., to $[2,3,10,20,23]$ for proper orthogonal decomposition and to [4, 18, 25, 29] for balanced truncation.

Proper orthogonal decomposition is based on projecting the dynamical system onto subspaces of basis elements that express characteristics of the expected solution. This is in contrast to, e.g., finite element techniques, where the elements are not correlated to the physical properties of the system they approximate.

In our present work, POD is applied to linear-quadratic optimal control problems. Linear-quadratic problems are interesting in several respects; in particular, they occur in each level of sequential quadratic programming (SQP) methods; see, e.g., $[22]$.

In contrast to methods of balanced truncation type, the POD method is somehow lacking a reliable a-priori error analysis. Unless its snapshots are generating a sufficiently rich state space, it is not a-priorily clear how far the optimal solution of the POD problem is from the exact one. On the other hand, the POD method is a universal tool that is applicable also to problems with time-dependent coefficients or to nonlinear equations. Moreover, by generating snapshots from the real (large)

Date: December 17, 2007.

2000 Mathematics Subject Classification. 35K90, 49K20, 65K05.

Key words and phrases. Optimal control, model reduction, proper orthogonal decomposition, a-posteriori error estimates.

The author S. V. gratefully acknowledges support by the Austrian Science Fund FWF under grant no. P19588-N18 and by te SFB Research Center "Mathematical Optimization in Biomedical Sciences" (SFB F32). 
model, a space is constructed that inhibits the main and relevant physical properties of the state system. This, and its ease of use makes POD very competitive in practical use, despite of a certain heuristic flavor.

In this paper, we again address the problem of error analysis. Our main focus is on an a-posteriori analysis. We use a fairly standard perturbation method to deduce how far the suboptimal control, computed on the basis of the POD model, is from the (unknown) exact one. This idea turned out to be very efficient in our examples. It is able to compensate for the lack of a priori analysis for POD methods. We also briefly discuss a priori error estimates. This analysis needs certain strong assumptions. Nevertheless, we include these results to show that there is a real chance to decrease the error up to zero by taking more snapshots, provided the assumptions are fulfilled.

In contrast to [15] the POD basis will be fixed during the numerical algorithm. Only the number of the utilized POD ansatz functions is increased, if necessary.

The paper is organized as follows: In Section 2, we introduce the linear-quadratic optimal control problem of parabolic type and review first-order necessary optimality conditions. The a-posteriori error analysis is carried out in Section 3, and the POD method is explained in Section 4. Moreover, an associated convergence analysis is carried out there. In Section 5, numerical test examples are presented.

\section{The linear-quadratic parabolic optimal control problem}

In this section, we introduce a class of linear-quadratic parabolic optimal control problems and recall the associated first-order necessary optimality conditions.

2.1 Problem formulation. Let $V$ and $H$ be real, separable Hilbert spaces and suppose that $V$ is dense in $H$ with compact embedding. By $\langle\cdot, \cdot\rangle_{H}$ we denote the inner product in $H$. The inner product in $V$ is given by a symmetric bounded, coercive, bilinear form $a: V \times V \rightarrow \mathbb{R}$ :

$$
\langle\varphi, \psi\rangle_{V}=a(\varphi, \psi) \quad \text { for all } \varphi, \psi \in V
$$

with associated norm $\|\cdot\|_{V}=\sqrt{a(\cdot, \cdot)}$. By identifying $H$ and its dual $H^{\prime}$ it follows that $V \hookrightarrow H=H^{\prime} \hookrightarrow V^{\prime}$, each embedding being continuous and dense.

Recall that for $T>0$ the space $W(0, T)$

$$
W(0, T)=\left\{\varphi \in L^{2}(0, T ; V): \varphi_{t} \in L^{2}\left(0, T ; V^{\prime}\right)\right\}
$$

is a Hilbert space endowed with the common inner product (see, for example, [6, p. 473]). It is well-known that $W(0, T)$ is continuously embedded into $C([0, T] ; H)$, the space of continuous functions from $[0, T]$ to $H$.

Let $\mathcal{J}$ be an open and bounded subset of $\mathbb{R}^{d}$ with $d \in \mathbb{N}$. By $U_{\text {ad }} \subset L^{2}(\mathcal{J})$ we define the closed, convex and bounded subset

$$
U_{\text {ad }}=\left\{u \in L^{2}(\mathcal{J}) \mid u_{a}(s) \leq u(s) \leq u_{b}(s) \text { for almost all (f.a.a.) } s \in \mathcal{J}\right\}
$$

with $u_{a}, u_{b} \in L^{2}(\mathcal{J})$ satisfying $u_{a} \leq u_{b}$ almost everywhere (a.e.) in J. For $y_{0} \in H$, $r \in L^{2}\left(0, T ; V^{\prime}\right)$ and $u \in U_{\text {ad }}$ we consider the linear evolution problem

$$
\begin{aligned}
\frac{\mathrm{d}}{\mathrm{d} t}\langle y(t), \varphi\rangle_{H}+a(y(t), \varphi) & =\langle(r+\mathcal{B} u)(t), \varphi\rangle_{V^{\prime}, V} & & \text { f.a.a. } t \in[0, T], \forall \varphi \in V, \\
\langle y(0), \varphi\rangle_{H} & =\left\langle y_{0}, \varphi\right\rangle_{H} & & \forall \varphi \in V,
\end{aligned}
$$

where $\mathcal{B}: L^{2}(\mathcal{J}) \rightarrow L^{2}\left(0, T ; V^{\prime}\right)$ is a continuous, linear operator. 
Example 2.1. Let us present an example for (2.2). Suppose that $\Omega \subset \mathbb{R}^{d}, d \in$ $\{1,2,3\}$, is an open and bounded domain with Lipschitz-continuous boundary $\Gamma=$ $\partial \Omega$. The boundary is split into two measurable disjoint parts $\Gamma_{C} \subset \Gamma$ and $\Gamma_{N}=$ $\Gamma \backslash \Gamma_{C}$. For $T>0$ we set $Q=(0, T) \times \Omega, \Sigma=(0, T) \times \Gamma, \Sigma_{C}=(0, T) \times \Gamma_{C}$ and $\Sigma_{N}=(0, T) \times \Gamma_{N}$. Let $H=L^{2}(\Omega), V=H^{1}(\Omega)$ and $\mathcal{J}=(0, T)$. Then, for given control $u \in L^{2}(0, T)$ we consider the linear heat equation

$$
y_{t}(t, \mathbf{x})-\Delta y(t, \mathbf{x})+y(t, \mathbf{x})=f(t, \mathbf{x}) \quad \text { f.a.a. }(t, \mathbf{x}) \in Q
$$

together with the inhomogeneous Neumann boundary condition

$$
\begin{array}{ll}
\frac{\partial y}{\partial n}(t, \mathbf{s})=u(t) b(t, \mathbf{s}) & \text { f.a.a. }(t, \mathbf{s}) \in \Sigma_{C}, \\
\frac{\partial y}{\partial n}(t, \mathbf{s})=g(t, \mathbf{x}) & \text { f.a.a. }(t, \mathbf{s}) \in \Sigma_{N}
\end{array}
$$

and with the initial condition

$$
y(0, \mathbf{x})=y_{0}(\mathbf{x}) \quad \text { f.a.a. } \mathbf{x} \in \Omega,
$$

where $y_{0} \in H$ is given. In (2.3) we suppose that $f \in L^{2}\left(0, T ; V^{\prime}\right), g \in L^{2}\left(\Sigma_{C}\right)$, $b \in L^{\infty}\left(0, T ; L^{2}\left(\Gamma_{C}\right)\right)$, and $y_{0} \in H$. Introducing the bilinear form $a: V \times V \rightarrow \mathbb{R}$ by

$$
a(\varphi, \psi)=\int_{\Omega} \nabla \varphi \cdot \nabla \psi+\varphi \psi \mathrm{d} \mathbf{x} \quad \text { for } \varphi, \psi \in V
$$

the linear, bounded functional $r \in L^{2}\left(0, T ; V^{\prime}\right)$ by

$$
\langle r(t), \phi\rangle_{V^{\prime}, V}=\int_{\Omega} f(t, \cdot) \phi \mathrm{d} \mathbf{x}+\int_{\Gamma_{N}} g(t, \cdot) \phi \mathrm{d} \mathbf{s} \quad \text { for } \phi \in V, t \in(0, T) \text { a.e. }
$$

and $\mathcal{B}: L^{2}(0, T) \rightarrow L^{2}\left(0, T ; V^{\prime}\right)$ as

$$
\langle(\mathcal{B} u)(t), \phi\rangle_{V^{\prime}, V}=u(t) \int_{\Gamma_{C}} b(t, \cdot) \mathrm{d} \mathbf{s} \quad \text { for } \phi \in V, t \in(0, T) \text { a.e. }
$$

it follows that the weak formulation of (2.3) can be expressed in the form (2.2). $\diamond$

It is well-known (see, e.g., [6]) that for every $r \in L^{2}\left(0, T ; V^{\prime}\right), u \in L^{2}(\mathcal{J})$ and $y_{0} \in H$ there exists a unique weak solution $y \in W(0, T)$ satisfying $(2.2)$ and

$$
\|y\|_{W(0, T)} \leq C\left(\|u\|_{L^{2}(\mathcal{J})}+\left\|y_{0}\right\|_{H}+\|r\|_{L^{2}\left(0, T ; V^{\prime}\right)}\right)
$$

with a constant $C>0$ independent of $y$.

Remark 2.2. Let $\hat{y}_{0} \in W(0, T)$ be the unique solution to

$$
\begin{aligned}
\frac{\mathrm{d}}{\mathrm{d} t}\left\langle\hat{y}_{0}(t), \varphi\right\rangle_{H}+a\left(\hat{y}_{0}(t), \varphi\right) & =\langle r(t), \varphi\rangle_{V^{\prime}, V} & & \text { f.a.a. } t \in[0, T], \forall \varphi \in V, \\
\left\langle\hat{y}_{0}(0), \varphi\right\rangle_{H} & =\left\langle y_{0}, \varphi\right\rangle_{H} & & \forall \varphi \in V .
\end{aligned}
$$

Moreover, we introduce the linear and bounded operator $\mathcal{S}: L^{2}(\mathcal{J}) \rightarrow W(0, T)$ as follows: $\tilde{y}=\mathcal{S} u \in W(0, T)$ is the unique solution to

$$
\begin{aligned}
\frac{\mathrm{d}}{\mathrm{d} t}\langle\tilde{y}(t), \varphi\rangle_{H}+a(\tilde{y}(t), \varphi) & =\langle(\mathcal{B} u)(t), \varphi\rangle_{V^{\prime}, V} & & \text { f.a.a. } t \in[0, T], \forall \varphi \in V, \\
\langle\tilde{y}(0), \varphi\rangle_{H} & =0 & & \forall \varphi \in V .
\end{aligned}
$$

Then, $y=\hat{y}_{0}+\mathcal{S} u$ is the weak solution to $(2.2)$. 
Next we introduce the cost functional $J: W(0, T) \times L^{2}(\mathcal{J}) \rightarrow \mathbb{R}$ by

$$
J(y, u)=\frac{\alpha_{1}}{2}\left\|\mathcal{C} y-z_{1}\right\|_{W_{1}}^{2}+\frac{\alpha_{2}}{2}\left\|\mathcal{D} y(T)-z_{2}\right\|_{W_{2}}^{2}+\frac{\sigma}{2}\|u\|_{L^{2}(\mathcal{J})}^{2},
$$

where $W_{1}, W_{2}$ are Hilbert spaces, $\mathcal{C}: L^{2}(0, T ; H) \rightarrow W_{1}$ and $\mathcal{D}: H \rightarrow W_{2}$ are bounded linear operators, and $\left(z_{1}, z_{2}\right) \in W_{1} \times W_{2}$ holds. Furthermore, $\alpha_{1}, \alpha_{2}$ are nonnegative parameters and $\sigma>0$.

Remark 2.3. In the context of Example 2.3 we choose $\alpha_{1}=0, \alpha_{2}=1, W_{2}=$ $L^{2}(\Omega), z_{2} \in L^{2}(\Omega), \mathcal{D}=$ id auf $L^{2}(\Omega)$, and $\mathcal{J}=(0, T)$. Then, $(2.5)$ yields the cost functional

$$
J(y, u)=\int_{\Omega}\left(y(T, \mathbf{x})-z_{2}(\mathbf{x})\right)^{2} \mathrm{~d} \mathbf{x}+\frac{\sigma}{2} \int_{0}^{T}(u(t))^{2} \mathrm{~d} t
$$

for $(y, u) \in W(0, T) \times L^{2}(\mathcal{J})$.

The optimal control problem is given by

$$
\min J(y, u) \quad \text { s.t. } \quad(y, u) \in W(0, T) \times U_{\text {ad }} \text { solves }(2.2) .
$$

Applying standard arguments (see [19], for instance) one can prove that there exists a unique optimal solution $\bar{x}=(\bar{y}, \bar{u})$ to $(\mathbf{P})$.

2.2 First-order optimality conditions. First-order necessary optimality conditions for our parabolic optimal control problem are well known. We briefly recall them, because they are needed for our subsequent error analysis.

Suppose that $\bar{x}=(\bar{y}, \bar{u})$ is the optimal solution to $(\mathbf{P})$ (in the paper, a bar indicates optimality). Then there exists a unique Lagrange-multiplier $\bar{p} \in W(0, T)$ satisfying together with $\bar{x}$ the first-order necessary optimality conditions, which consist of the state equations (2.2), the adjoint equations in $[0, T]$

$$
\begin{aligned}
-\frac{\mathrm{d}}{\mathrm{d} t}\langle\bar{p}(t), \varphi\rangle_{H}+a(\bar{p}(t), \varphi) & =\alpha_{1}\left\langle z_{1}-\mathcal{C} \bar{y}, \mathcal{C} \varphi\right\rangle_{W_{1}} \text { f.a.a. } t \in[0, T], \forall \varphi \in V, \\
\langle\bar{p}(T), \varphi\rangle_{H} & =\alpha_{2}\left\langle z_{2}-\mathcal{D} \bar{y}(T), \mathcal{D} \varphi\right\rangle_{W_{2}} \quad \forall \varphi \in V
\end{aligned}
$$

and of the variational inequality

$$
\left\langle\sigma \bar{u}-\mathcal{B}^{\star} \bar{p}, u-\bar{u}\right\rangle_{L^{2}(\mathcal{J})} \geq 0 \quad \forall u \in \mathcal{U}_{\mathrm{ad}} .
$$

Here, the linear and bounded operator $\mathcal{B}^{\star}: L^{2}(0, T ; V) \rightarrow L^{2}(\mathcal{J})^{\prime} \sim L^{2}(\mathcal{J})$ stands for the dual operator of $\mathcal{B}$ satisfying

$$
\langle\mathcal{B} u, \varphi\rangle_{L^{2}\left(0, T ; V^{\prime}\right), L^{2}(0, T ; V)}=\left\langle\mathcal{B}^{\star} \varphi, u\right\rangle_{L^{2}(\mathcal{J})} \quad \forall(u, \varphi) \in L^{2}(\mathcal{J}) \times L^{2}(0, T ; V) .
$$

In Remark 2.2 the linear and bounded operator $\mathcal{S}$ has been defined. The associated dual $\mathcal{S}^{\star}: W(0, T)^{\prime} \rightarrow L^{2}(\mathcal{J})$ is defined as

$$
\left\langle\mathcal{S}^{\star} f, u\right\rangle_{L^{2}(\mathcal{J})}=\langle f, \mathcal{S} u\rangle_{W(0, T)^{\prime}, W(0, T)} \quad \forall(f, u) \in W(0, T)^{\prime} \times L^{2}(\mathcal{J}) .
$$

We will make use of the following lemma that is a variant of Lemma 4.1 in [12]. For its proof we refer the reader to the Appendix.

Lemma 2.4. Suppose that $z_{1} \in L^{2}(0, T ; H), z_{2} \in H$ and $\bar{y}=\hat{y}_{0}+\mathcal{S} \bar{u} \in W(0, T)$ with given optimal control $\bar{u} \in L^{2}(\mathcal{J})$, where $\hat{y}_{0}$ has been defined in Remark 2.2 . Moreover, let $\bar{p} \in W(0, T)$ denote the unique solution to the adjoint system (2.6). 
Furthermore, $\Theta: W(0, T) \rightarrow W(0, T)^{\prime}$ and $\Xi: L^{2}(0, T ; H) \times H \rightarrow W(0, T)^{\prime}$ are defined by

$$
\begin{gathered}
\langle\Theta(\chi), \phi\rangle_{W(0, T)^{\prime}, W(0, T)}=\alpha_{1}\langle\mathcal{C} \chi, \mathcal{C} \phi\rangle_{W_{1}}+\alpha_{2}\langle\mathcal{D} \chi(T), \mathcal{D} \phi(T)\rangle_{W_{2}}, \\
\left\langle\Xi\left(\chi_{1}, \chi_{2}\right), \varphi\right\rangle_{W(0, T)^{\prime}, W(0, T)}=\alpha_{1}\left\langle\chi_{1}, \mathcal{C} \varphi\right\rangle_{W_{1}}+\alpha_{2}\left\langle\chi_{2}, \mathcal{D} \varphi(T)\right\rangle_{W_{2}} \\
\text { for } \chi, \phi, \varphi \in W(0, T) \text { and }\left(\chi_{1}, \chi_{2}\right) \in W_{1} \times W_{2} . \text { Then it follows that }
\end{gathered}
$$

$$
\mathcal{B}^{\star} \bar{p}=\mathcal{S}^{\star}\left(\Xi\left(z_{1}, z_{2}\right)-\Theta(\bar{y})\right) \in L^{2}(\mathcal{J}) .
$$

Remark 2.5. We continue the discussion of Example 2.3 and Remark 2.3. The adjoint equations (2.6) are given by

$$
\begin{aligned}
-\bar{p}_{t}(t, \mathbf{x})-\Delta \bar{p}(t, \mathbf{x})+\bar{p}(t, \mathbf{x}) & =0 & & \text { f.a.a. }(t, \mathbf{x}) \in Q, \\
\frac{\partial \bar{p}}{\partial n}(t, \mathbf{s}) & =0 & & \text { f.a.a. }(t, \mathbf{s}) \in \Sigma, \\
p(T, \mathbf{x}) & =z_{2}(\mathbf{x})-\bar{y}(T, \mathbf{x}) & & \text { f.a.a. } \mathbf{x} \in \Omega .
\end{aligned}
$$

Moreover, the variational inequality (2.7) has the form

$$
\int_{0}^{T}\left(\sigma \bar{u}(t)-\int_{\Gamma_{C}} b(t, \mathbf{s}) \bar{p}(t, \mathbf{s}) \mathrm{d} \mathbf{s}\right)(u(t)-\bar{u}(t)) \mathrm{d} t \geq 0 \quad \text { for all } u \in U_{\mathrm{ad}}
$$

and $\mathcal{B}^{\star} \bar{p} \in L^{2}(\mathcal{J})$ is given by $\left(\mathcal{B}^{\star} \bar{p}\right)(t)=\int_{\Gamma_{C}} b(t, \mathbf{s}) \bar{p}(t, \mathbf{s})$ ds f.a.a. $t \in[0, T]$.

2.3 The reduced control problem. Utilizing the solution operator $\mathcal{S}$ (see Remark 2.2) we introduce the so-called reduced cost functional as

$$
\hat{J}(u)=J\left(\hat{y}_{0}+\mathcal{S} u, u\right) .
$$

Then, we can express $(\mathbf{P})$ as the reduced problem

$$
\min \hat{J}(u) \quad \text { s.t. } \quad u \in U_{\text {ad }}
$$

It follows that $\hat{J}^{\prime}(\bar{u})=\sigma \bar{u}-\mathcal{B}^{\star} \bar{p} \in L^{2}(\mathcal{J})$ is the gradient of $\hat{J}$ at $\bar{u}$, where $\bar{p}$ solves the dual sytem (2.6) for $\bar{y}=\hat{y}_{0}+\mathcal{S} \bar{u}$. Moreover, the variational inequality (2.7) is equivalent to

$$
\bar{u}(s)=\mathcal{P}_{\left[u_{a}(s), u_{b}(s)\right]}\left(\frac{1}{\sigma}\left(\mathcal{B}^{\star} \bar{p}\right)(s)\right) \text { f.a.a. } s \in \mathcal{J},
$$

where $\mathcal{P}_{[a, b]}: \mathbb{R} \rightarrow[a, b]$ denotes the projection operator onto the convex interval $[a, b] \subset \mathbb{R}$.

\section{A-posteriori error analysis}

In principle, this section contains the main idea underlying our a-posteriori error analysis. Suppose that $u_{p}$ is an arbitrary control of $U_{\mathrm{ad}}$. Our goal is to estimate the difference

$$
\left\|\bar{u}-u_{p}\right\|_{L^{2}(\mathcal{J})}
$$

without the knowledge of the optimal solution $\bar{u}$. The associated idea is not new. For instance, it was used by Malanowski et al. [21] in the context of error estimates for the optimal control of ODEs. It was extended later to elliptic optimal control problems in [1] and [5]. Let us explain this basic idea here. 
If $u_{p} \neq \bar{u}$ then $u_{p}$ does not satisfy the necessary (and by convexity sufficient) optimality conditions (2.7) respectively (2.11). However, there exists a function $\zeta \in L^{2}(\mathcal{J})$ such that

$$
\left\langle\sigma u_{p}-\mathcal{B}^{\star} p_{p}+\zeta, u-u_{p}\right\rangle_{L^{2}(\mathcal{J})} \geq 0 \quad \forall u \in U_{a d},
$$

where $p_{p} \in W(0, T)$ solves the adjoint equation associated with $u_{p}$

$$
\begin{aligned}
-\frac{\mathrm{d}}{\mathrm{d} t}\left\langle p_{p}(t), \varphi\right\rangle_{H}+a\left(p_{p}(t), \varphi\right) & =\alpha_{1}\left\langle z_{1}-\mathcal{C} y_{p}, \mathcal{C} \varphi\right\rangle_{W_{1}} \text { f.a.a. } t \in[0, T], \forall \varphi \in V, \\
\left\langle p_{p}(T), \varphi\right\rangle_{H} & =\alpha_{2}\left\langle z_{2}-\mathcal{D} y_{p}(T), \mathcal{D} \varphi\right\rangle_{W_{2}} \quad \forall \varphi \in V,
\end{aligned}
$$

and $y_{p}=\hat{y}+\mathcal{S} u_{p}$ is the state corresponding to $u_{p}$. Therefore, $u_{p}$ satisfies the optimality condition of a perturbed parabolic optimal control problem with "perturbation" $\zeta$. We refer, e.g., to [1]. The smaller $\zeta$ is, the closer $u_{p}$ is to $\bar{u}$.

The computation of $\zeta$ is possible on the basis of the known data $u_{p}, y_{p}$, and $p_{p}$. We carry out this construction below in Proposition 3.2. First, however, we estimate $\left\|\bar{u}-u_{p}\right\|_{L^{2}(\mathcal{J})}$ in terms of $\|\zeta\|_{L^{2}(\mathcal{J})}$. Choosing $u=u_{p}$ in (2.7) and $u=\bar{u}$ in (3.1) we obtain

$$
\begin{aligned}
0 & \leq\left\langle\sigma \bar{u}-\mathcal{B}^{\star} \bar{p}, u_{p}-\bar{u}\right\rangle_{L^{2}(\mathcal{J})}+\left\langle\sigma u_{p}-\mathcal{B}^{\star} p_{p}+\zeta, \bar{u}-u_{p}\right\rangle_{L^{2}(\mathcal{J})} \\
& =\left\langle\sigma \bar{u}-\mathcal{B}^{\star} \bar{p}, u_{p}-\bar{u}\right\rangle_{L^{2}(\mathcal{J})}-\left\langle\sigma u_{p}-\mathcal{B}^{\star} p_{p}+\zeta, u_{p}-\bar{u}\right\rangle_{L^{2}(\mathcal{J})} \\
& =\sigma\left\langle\bar{u}-u_{p}, u_{p}-\bar{u}\right\rangle_{L^{2}(\mathcal{J})}-\left\langle\mathcal{B}^{\star}\left(\bar{p}-p_{p}\right), u_{p}-\bar{u}\right\rangle_{L^{2}(\mathcal{J})}-\left\langle\zeta, u_{p}-\bar{u}\right\rangle_{L^{2}(\mathcal{J})} \\
& =-\sigma\left\|\bar{u}-u_{p}\right\|_{L^{2}(\mathcal{J})}^{2}+\left\langle\mathcal{B}^{\star}\left(p_{p}-\bar{p}\right), u_{p}-\bar{u}\right\rangle_{L^{2}(\mathcal{J})}-\left\langle\zeta, u_{p}-\bar{u}\right\rangle_{L^{2}(\mathcal{J})} .
\end{aligned}
$$

Lemma 2.4 yields

$$
\mathcal{B}^{\star} \bar{p}=\mathcal{S}^{\star}\left(\Xi\left(z_{1}, z_{2}\right)-\Theta(\bar{y})\right) \quad \text { with } \bar{y}=\hat{y}_{0}+\mathcal{S} \bar{u} .
$$

Analogously, we obtain

$$
\mathcal{B}^{\star} p_{p}=\mathcal{S}^{\star}\left(\Xi\left(z_{1}, z_{2}\right)-\Theta\left(y_{p}\right)\right) \quad \text { with } y_{p}=\hat{y}_{0}+\mathcal{S} u_{p} .
$$

Thus,

$$
\begin{aligned}
\left\langle\mathcal{B}^{\star}(\right. & \left.\left.p_{p}-\bar{p}\right), u_{p}-\bar{u}\right\rangle_{L^{2}(\mathcal{J})} \\
& =\left\langle\mathcal{S}^{\star}\left(\Xi\left(z_{1}, z_{2}\right)-\Theta\left(y_{p}\right)\right)-\mathcal{S}^{\star}\left(\Xi\left(z_{1}, z_{2}\right)-\Theta(\bar{y})\right), u_{p}-\bar{u}\right\rangle_{L^{2}(\mathcal{J})} \\
& =\left\langle\Theta(\bar{y})-\Theta\left(y_{p}\right), \mathcal{S}\left(u_{p}-\bar{u}\right)\right\rangle_{L^{2}(\mathcal{J})}=-\left\langle\Theta\left(y_{p}-\bar{y}\right), y_{p}-\bar{y}\right\rangle_{L^{2}(\mathcal{J})} \\
& =-\alpha_{1}\left\|\mathcal{C}\left(y_{p}-\bar{y}\right)\right\|_{W_{1}}^{2}-\alpha_{2}\left\|\mathcal{D}\left(y_{p}-\bar{y}\right)\right\|_{W_{2}}^{2} \leq 0 .
\end{aligned}
$$

Therefore we conclude from (3.3) that

$$
\sigma\left\|\bar{u}-u_{p}\right\|_{L^{2}(\mathcal{J})}^{2} \leq-\left\langle\zeta, u_{p}-\bar{u}\right\rangle_{L^{2}(\mathcal{J})} \leq\|\zeta\|_{L^{2}(\mathcal{J})}\left\|\bar{u}-u_{p}\right\|_{L^{2}(\mathcal{J})},
$$

which gives easily

$$
\left\|\bar{u}-u_{p}\right\|_{L^{2}(\mathcal{J})} \leq \frac{1}{\sigma}\|\zeta\|_{L^{2}(\mathcal{J})} .
$$

Summarizing we have proved the following theorem.

Theorem 3.1. Let $\bar{u}$ be the optimal solution to $(\mathbf{P}), \bar{y}$ the associated optimal state, and $\bar{p}$ the associated Lagrange multiplier. Suppose that $u_{p} \in U_{\text {ad }}$ is chosen arbitrarily, $y_{p}=\hat{y}+\mathcal{S} u_{p}$, and $p_{p}$ is the solution to (3.2). Then it follows that

$$
\left\|\bar{u}-u_{p}\right\|_{L^{2}(\mathcal{J})} \leq \frac{1}{\sigma}\|\zeta\|_{L^{2}(\mathcal{J})}
$$


where $\zeta$ is chosen such that (3.1) holds.

We proceed by constructing the function $\zeta$. Suppose that we have $u_{p}$ and the associated adjoint state $p_{p}$ solving to (3.2). The goal is to determine $\zeta \in L^{2}(\mathcal{J})$ satisfying (3.1). We distinguish between three different cases.

1) Case $u_{p}(s)=u_{a}(s)$ for fixed $s \in \mathcal{J}$ : Then, $u(s)-u_{p}(s)=u(s)-u_{a}(s) \geq 0$ for all $u \in U_{\text {ad. }}$. Hence, $\zeta(s)$ has to satisfy

$$
\left(\sigma u_{p}-\mathcal{B}^{\star} p_{p}\right)(s)+\zeta(s) \geq 0 .
$$

Setting

$$
\begin{aligned}
\zeta(s) & =\left[\left(\sigma u_{p}-\mathcal{B}^{\star} p_{p}\right)(s)\right]_{-}=-\min \left(0,\left(\sigma u_{p}-\mathcal{B}^{\star} p_{p}\right)(s)\right) \\
& =\frac{1}{2}\left(\left|\left(\sigma u_{p}-\mathcal{B}^{\star} p_{p}\right)(s)\right|-\left(\sigma u_{p}-\mathcal{B}^{\star} p_{p}\right)(s)\right)
\end{aligned}
$$

the value $\zeta(s)$ satisfies (3.4). Here, $[s]_{-}=-\min (0, s)$ denotes the negative part function.

2) Case $u_{p}(s)=u_{b}(s)$ for fixed $s \in \mathrm{J}$ : Now, $u(s)-u_{p}(s)=u(s)-u_{b}(s) \leq 0$ for all $u \in U_{\text {ad. }}$. Analogously to the first case we define

$$
\begin{aligned}
\zeta(s) & =\left[\left(\sigma u_{p}-\mathcal{B}^{\star} p_{p}\right)(s)\right]_{+}=\max \left(0,\left(\sigma u_{p}-\mathcal{B}^{\star} p_{p}\right)(s)\right) \\
& =\frac{1}{2}\left(\left(\sigma u_{p}-\mathcal{B}^{\star} p_{p}\right)(s)+\left|\left(\sigma u_{p}-\mathcal{B}^{\star} p_{p}\right)(s)\right|\right)
\end{aligned}
$$

to ensure (3.4), where, $[s]_{+}=\max (0, s)$ denotes the positive part function.

3) Case $u_{a}(s)<u_{p}(s)<u_{b}(s)$ for fixed $s \in \mathcal{J}$ : Consequently, $\left(\sigma u_{p}-\mathcal{B}^{\star} p_{p}\right)(s)+$ $\zeta(s)=0$ holds so that $\zeta(s)=-\left(\sigma u_{p}-\mathcal{B}^{\star} p_{p}\right)(s)$ guarantees (3.4).

Clearly, $\zeta \equiv 0$ holds in the case, where $u_{p}$ satisfies the first-order necessary optimality conditions.

Proposition 3.2. Suppose that the hypotheses of Theorem 3.1 are satisfied. Define $\zeta \in L^{2}(\mathcal{J})$ as follows:

$$
\zeta(s)= \begin{cases}{\left[\left(\sigma u_{p}-\mathcal{B}^{\star} p_{p}\right)(s)\right]_{-}} & \text {on } \mathcal{A}_{-}=\left\{s \in \mathcal{J} \mid u_{p}(s)=u_{a}(s)\right\}, \\ {\left[\left(\sigma u_{p}-\mathcal{B}^{\star} p_{p}\right)(s)\right]_{+}} & \text {on } \mathcal{A}_{+}=\left\{s \in \mathcal{J} \mid u_{p}(s)=u_{b}(s)\right\}, \\ -\left(\sigma u_{p}-\mathcal{B}^{\star} p_{p}\right)(s) & \text { on } \mathcal{J}=\mathcal{J} \backslash\left(\mathcal{A}_{-} \cup \mathcal{A}_{+}\right) .\end{cases}
$$

Then, the estimate

$$
\left\|\bar{u}-u_{p}\right\|_{L^{2}(\mathcal{J})} \leq \frac{1}{\sigma}\|\zeta\|_{L^{2}(\mathcal{J})}
$$

is satisfied.

We will call (3.6) an a-posteriori error estimate, since, in the next section, we shall apply it to suboptimal controls $u_{p}$ that have already been computed from a POD model. After having computed $u_{p}$, we determine the associated state $y_{p}$ and adjoint state (Lagrange multiplier) $p_{p}$. Then we can determine $\zeta$ and its $L^{2}$-norm and (3.6) gives an upper bound for the distance of $u_{p}$ to $\bar{u}$. In this way, the error caused by the POD method can be estimated a-posteriorily. If the error is too large, then we have to include more POD basis functions in our Galerkin ansatz; see Section 4.6. This approach compensates the lack of a-priori error estimates for the POD method. 
Remark 3.3. Similar arguments can be used to derive an analogous error estimate (as in (3.6)) for linear-quadratic optimal control problems governed by linear elliptic problems; see Section 5, Run 2.

\section{The POD Galerkin discretization}

In this section we briefly introduce the POD method and derive the reduced-order model. To keep the notation simple, we apply only a spatial discretization with POD basis functions, but no time integration by, e.g., an implicit Euler method. Therefore, in the analysis we utilize a continuous POD. Let us mention the work [8], where convergence of POD Galerkin approximations for evolution problems is analyzed using also a continuous version of POD.

4.1 The POD method. Let an arbitrary $u \in L^{2}(\mathcal{J})$ be chosen such that the corresponding state variable $y=\hat{y}_{0}+\mathcal{S} u \in W(0, T)$ belongs to $C([0, T] ; V)$. Then,

$$
\mathcal{V}=\operatorname{span}\{y(t) \mid t \in[0, T]\} \subseteq V .
$$

If $y_{0} \neq 0$ holds, then $\operatorname{span}\left\{y_{0}\right\} \subset \mathcal{V}$ and $d=\operatorname{dim} \mathcal{V} \geq 1$, but $\mathcal{V}$ may have infinite dimension. We define a bounded linear operator $\mathcal{Y}: L^{2}(0, T) \rightarrow V$ by

$$
\mathcal{Y} \varphi=\int_{0}^{T} \varphi(t) y(t) \mathrm{d} t \quad \text { for } \varphi \in L^{2}(0, T) .
$$

Its Hilbert space adjoint $\mathcal{Y}^{\star}: V \rightarrow L^{2}(0, T)$ satisfying

$$
\langle\mathcal{Y} \varphi, z\rangle_{V}=\left\langle\varphi, \mathcal{Y}^{\star} z\right\rangle_{L^{2}(0, T)} \quad \text { for }(\varphi, z) \in L^{2}(0, T) \times V
$$

is given by

$$
\left(\mathcal{Y}^{\star} z\right)(t)=\langle z, y(t)\rangle_{V} \quad \text { for } z \in V \text { and f.a.a. } t \in[0, T] .
$$

The bounded linear operator $\mathcal{R}=\mathcal{Y Y}^{\star}: V \rightarrow \mathcal{V} \subseteq V$ has the form

$$
\mathcal{R} z=\int_{0}^{T}\langle z, y(t)\rangle_{V} y(t) \mathrm{d} t \quad \text { for } z \in V .
$$

Moreover, let $\mathcal{K}=\mathcal{Y}^{\star} \mathcal{Y}: L^{2}(0, T) \rightarrow L^{2}(0, T)$ be defined by

$$
(\mathcal{K} \varphi)(t)=\int_{0}^{T}\langle y(s), y(t)\rangle_{V} \varphi(s) \mathrm{d} s \quad \text { for } \varphi \in L^{2}(0, T) .
$$

First we observe that the linear and bounded operator $\mathcal{R}$ is self-adjoint. Since $y \in W(0, T) \subset L^{2}(0, T ; V)$ the kernel of $\mathcal{K}$ is square integrable over $(0, T) \times(0, T)$, so that the integral operator is Hilbert-Schmidt and therefore compact. This implies that $\mathcal{R}$ is compact as well. Moreover, $\mathcal{R}$ is non-negative. From the Hilbert-Schmidt theorem $\left[24\right.$, p. 203] it follows that there exists a complete orthonormal basis $\left\{\psi_{i}\right\}_{i=1}^{d}$ for $\mathcal{V}=$ range $(\mathcal{R})$ and a sequence $\left\{\lambda_{i}\right\}_{i=1}^{d}$ of real numbers such that

$$
\mathcal{R} \psi_{i}=\lambda_{i} \psi_{i} \text { for } i=1, \ldots, d \quad \text { and } \quad \lambda_{1} \geq \lambda_{2} \geq \ldots \geq \lambda_{d} \geq 0 .
$$

Remark 4.1. $\quad 1$ ) By the Riesz-Schauder theorem the spectrum of $\mathcal{R}$ is a pure point spectrum except for possibly 0; see [24, p. 203].

2) To obtain a complete orthonormal basis in the separable Hilbert space $V$ we need an orthonormal basis for $(\operatorname{range}(\mathcal{R}))^{\perp}$. This can be done by the Gram-Schmidt procedure. Hence, we suppose in the following that $\left\{\psi_{i}\right\}_{i=1}^{\infty}$ is a complete orthonormal basis for $V$. 
3) If $1 \leq d=\operatorname{dim} \mathcal{V}<\infty$ holds and $\left\{\psi_{i}\right\}_{i=1}^{\infty}$ is as described in Part 2), it follows that $\lambda_{i}>0$ for $1 \leq i \leq d$ and $\mathcal{R} \psi_{i}=0$ for all $i>d$.

4) Analogously to the theory of singular value decompositions for matrices, we find that the linear, bounded, compact and self-adjoint operator $\mathcal{K}$ has the same eigenvalues $\left\{\lambda_{i}\right\}_{i \in \mathbb{N}}$ as the operator $\mathcal{R}$. For all $\lambda_{i}>0$ the corresponding eigenfunctions of $\mathcal{K}$ are given by

$$
v_{i}(t)=\frac{1}{\sqrt{\lambda_{i}}}\left(\mathcal{Y}^{*} \psi_{i}\right)(t)=\frac{1}{\sqrt{\lambda_{i}}}\left\langle\psi_{i}, y(t)\right\rangle_{V} \text { f.a.a. } t \in[0, T] \text { and } 1 \leq i \leq \ell \text {. }
$$

In the following proposition we formulate properties of the eigenvalues and eigenfunctions of $\mathcal{R}$. Therefore, for given $\ell \in \mathbb{N}$ we introduce the mapping

$$
\mathfrak{J}: \underbrace{V \times \ldots \times V}_{\ell-\text { times }} \rightarrow \mathbb{R}, \quad \mathfrak{J}\left(\psi_{1}, \ldots, \psi_{\ell}\right):=\int_{0}^{T}\left\|y(t)-\sum_{i=1}^{\ell}\left\langle y(t), \psi_{i}\right\rangle_{V} \psi_{i}\right\|_{V}^{2} \mathrm{~d} t .
$$

Note that

$$
\mathfrak{J}\left(\psi_{1}, \ldots, \psi_{\ell}\right)=\int_{0}^{T}\left\|y(t)-\mathcal{P}^{\ell} y(t)\right\|_{V}^{2} \mathrm{~d} t .
$$

Proposition 4.2. Suppose that $V$ is a separable Hilbert space, $y \in C([0, T] ; V)$ holds and $\mathcal{V}$ is given as in (4.1). Let the linear operator $\mathcal{R}: V \rightarrow V$ be defined as in (4.2). Then, $\mathcal{R}$ is bounded, self-adjoint, compact and non-negative, and there exists $\left\{\lambda_{i}\right\}_{i \in \mathbb{N}}$ and $\left\{\psi_{i}\right\}_{i \in \mathbb{N}}$ satisfying (4.3). Moreover, for any $\ell \leq d=\operatorname{dim} \mathcal{V}$ the elements $\left\{\psi_{i}\right\}_{i=1}^{\ell}$ solve the minimization problem

$$
\min \mathfrak{J}\left(\tilde{\psi}_{1}, \ldots, \tilde{\psi}_{\ell}\right) \quad \text { s.t. }\left\langle\tilde{\psi}_{j}, \tilde{\psi}_{i}\right\rangle_{V}=\delta_{i j} \quad \text { for } 1 \leq i, j \leq \ell
$$

and

$$
\mathfrak{J}\left(\psi_{1}, \ldots, \psi_{\ell}\right)=\sum_{i=\ell+1}^{\infty} \lambda_{i} .
$$

For a proof we refer to [13, Section 3], [24, Sections II and VI] and [27], for instance.

4.2 The discrete POD method. In real computations, we do not have the whole trajectory $y(t)$ for all $t \in[0, T]$. For that purpose let $0=t_{1}<t_{2}<\ldots<t_{n}=T$ be a given grid in $[0, T]$ and let $y_{j}=y\left(t_{j}\right)$ denote approximations for $y$ at time instance $t_{j}, j=1, \ldots, n$. We set $\mathcal{V}^{n}=\operatorname{span}\left\{y_{1}, \ldots, y_{n}\right\}$ with $d^{n}=\operatorname{dim} \mathcal{V}^{n} \leq n$. Then, for given $\ell \leq n$ we consider the minimization problem

$$
\min \sum_{j=1}^{n} \alpha_{j}\left\|y_{j}-\sum_{i=1}^{\ell}\left\langle y_{j}, \psi_{i}^{n}\right\rangle_{V} \psi_{i}^{n}\right\|_{V}^{2} \text { s.t. }\left\langle\psi_{i}^{n}, \psi_{j}^{n}\right\rangle_{V}=\delta_{i j} \text { for } 1 \leq i, j \leq \ell
$$

instead of (4.5). In (4.7) the $\alpha_{j}$ 's stand for the trapezoidal weights

$$
\alpha_{1}=\frac{t_{2}-t_{1}}{2}, \quad \alpha_{j}=\frac{t_{j+1}-t_{j-1}}{2} \text { for } 2 \leq j \leq n-1, \quad \alpha_{n}=\frac{t_{n}-t_{n-1}}{2} .
$$

The solution to (4.7) is given by the solution to the eigenvalue problem

$$
\mathcal{R}^{n} \psi_{i}^{n}=\sum_{j=1}^{n} \alpha_{j}\left\langle y_{j}, \psi_{i}^{n}\right\rangle_{V} y_{j}=\lambda_{i}^{n} \psi_{i}^{n}, \quad i=1, \ldots, \ell
$$


where $\mathcal{R}^{n}: V \rightarrow \mathcal{V}^{n} \subset V$ is a linear, bounded, compact, self-adjoint and nonnegative operator. Thus, there exists an orthonormal set $\left\{\psi_{i}^{n}\right\}_{i=1}^{d^{n}}$ of eigenfunctions and corresponding non-negative eigenvalues $\left\{\lambda_{i}^{n}\right\}_{i=1}^{d^{n}}$ satisfying

$$
\mathcal{R}^{n} \psi_{i}^{n}=\lambda_{i}^{n} \psi_{i}^{n}, \quad \lambda_{1}^{n} \geq \lambda_{2}^{n} \geq \ldots \geq \lambda_{d^{n}}^{n}>0 .
$$

We refer to [17], where the relationship between (4.3) and (4.8) is investigated.

4.3 POD Galerkin scheme for the state equation. The analysis worked out in Sections 4.3-4.6 is not needed to understand the main principle of our a-posteriori error estimation. This has already been explained in the preceding section and the reader might proceed with Theorem 4.11. However, this analysis shows that there is a real chance to decrease the error by increasing the number of snapshots used by the POD method, provided that some natural assumptions are satisfied. First, we derive an error estimate for the state equation, where the control $u$ is fixed.

Let $y=\hat{y}_{0}+\mathcal{S} u$ be the state associated with some control $u \in L^{2}(\mathcal{J})$, and let $\mathcal{V}$ be given as in (4.1). We fix $\ell$ with $\ell \leq \operatorname{dim} \mathcal{V}$ and compute the first $\ell$ POD basis functions $\psi_{1}, \ldots, \psi_{\ell} \in V$ by solving either $\mathcal{R} \psi_{i}=\lambda_{i} \psi_{i}$ or $\mathcal{K} v_{i}=\lambda v_{i}$ for $i=1, \ldots, \ell$ (see Remark 4.1). Then we define the finite dimensional linear space

$$
V^{\ell}=\operatorname{span}\left\{\psi_{1}, \ldots, \psi_{\ell}\right\} \subset V \text {. }
$$

Endowed with the topology in $V$ it follows that $V^{\ell}$ is a Hilbert space. Let $\mathcal{P}^{\ell}$ denote the orthogonal projection $\mathcal{P}^{\ell}$ of $V$ onto $V^{\ell}$ defined by

$$
\mathcal{P}^{\ell} \varphi=\sum_{i=1}^{\ell}\left\langle\varphi, \psi_{i}\right\rangle_{V} \psi_{i} \quad \text { for } \varphi \in V
$$

Combining (4.4) and (4.5) we obtain that

$$
\mathfrak{J}\left(\psi_{1}, \ldots, \psi_{\ell}\right)=\int_{0}^{T}\left\|y(t)-\mathcal{P}^{\ell} y(t)\right\|_{V}^{2} \mathrm{~d} t=\left\|y-\mathcal{P}^{\ell} y\right\|_{L^{2}(0, T ; V)}^{2}=\sum_{i=\ell+1}^{\infty} \lambda_{i} .
$$

The POD Galerkin scheme for the state equation (2.2) leads to the following linear problem: determine a function $y^{\ell}=\sum_{i=1}^{\ell} y_{i}(t) \psi_{i}$ such that

$$
\begin{gathered}
\frac{\mathrm{d}}{\mathrm{d} t}\left\langle y^{\ell}(t), \psi\right\rangle_{H}+a\left(y^{\ell}(t), \psi\right)=\langle(r+\mathcal{B} u)(t), \psi\rangle_{V^{\prime}, V} \text { f.a.a. } t \in[0, T], \forall \psi \in V^{\ell}, \\
\left\langle y^{\ell}(0), \psi\right\rangle_{H}=\left\langle y_{0}, \psi\right\rangle_{H} \quad \forall \psi \in V^{\ell}
\end{gathered}
$$

For every $r \in L^{2}\left(0, T ; V^{\prime}\right), u \in L^{2}(\mathcal{J}), y_{0} \in H$ and for every $\ell \in \mathbb{N}$ problem (4.11) admits a unique solution $y^{\ell} \in H^{1}\left(0, T ; V^{\ell}\right)$; see [12, Proposition 3.4]. From $V^{\ell} \hookrightarrow V$ it follows that $y^{\ell} \in W(0, T)$ holds.

Let $\hat{y}_{0}^{\ell} \in H^{1}\left(0, T ; V^{\ell}\right)$ be the solution to (4.11) for $u \equiv 0$. Analogously to Remark 2.2 we introduce the linear operator $\mathcal{S}^{\ell}: L^{2}(\mathcal{J}) \rightarrow H^{1}\left(0, T ; V^{\ell}\right)$ for fixed $\ell$ : For given $u \in L^{2}(\mathcal{J})$ the element $\tilde{y}^{\ell}=\mathcal{S}^{\ell} u$ solves (4.11) with $r \equiv 0$ and $y_{0} \equiv 0$. Thus, $y^{\ell}$ is given by $y^{\ell}=\hat{y}_{0}^{\ell}+\tilde{y}^{\ell}$. It follows from [12, Proposition 3.4] that the operator $\mathcal{S}^{\ell}$ is bounded independently of $\ell$.

Proposition 4.3. For given $r \in L^{2}\left(0, T ; V^{\prime}\right), u \in L^{2}(\mathcal{J})$, and $y_{0} \in H$ we suppose that $y=\hat{y}+\mathcal{S} u$ belongs to $y \in C([0, T] ; V)$. Suppose that, for $\ell \leq \operatorname{dim} \mathcal{V}$, the 
elements $\left\{\psi_{i}\right\}_{i=1}^{\ell}$ solve (4.5). Then, there exists a constant $C>0$ such that

$$
\left\|y-y^{\ell}\right\|_{W(0, T)}^{2} \leq C\left(\left\|y^{\ell}(0)-\mathcal{P}^{\ell} y_{0}\right\|_{H}^{2}+\left\|y_{t}-\mathcal{P}^{\ell} y_{t}\right\|_{L^{2}\left(0, T ; V^{\prime}\right)}^{2}+\sum_{i=\ell+1}^{\infty} \lambda_{i}\right),
$$

where the linear projector $\mathcal{P}^{\ell}: V \rightarrow V^{\ell}$ is given by (4.9) and $y^{\ell}=\hat{y}_{0}^{\ell}+\mathcal{S}^{\ell} u$ denotes the unique solution to (4.11).

Proof. Proceeding similarly as in the proof of Proposition 4.7 in [12] it follows that

$$
\left\|y-y^{\ell}\right\|_{W(0, T)}^{2} \leq C\left(\left\|y^{\ell}(0)-\mathcal{P}^{\ell} y_{0}\right\|_{H}^{2}+\left\|y-\mathcal{P}^{\ell} y\right\|_{W(0, T)}^{2}\right)
$$

see in the Appendix. Utilizing

$$
\left\|y-\mathcal{P}^{\ell} y\right\|_{W(0, T)}^{2}=\left\|y-\mathcal{P}^{\ell} y\right\|_{L^{2}(0, T ; V)}^{2}+\left\|y_{t}-\mathcal{P}^{\ell} y_{t}\right\|_{L^{2}\left(0, T ; V^{\prime}\right)}^{2}
$$

and (4.10) we obtain the claim.

Proposition 4.3 permits to show that the POD approximations $y^{\ell}$ converge to $y$ in the $W(0, T)$-norm:

Proposition 4.4. For given $r \in L^{2}\left(0, T ; V^{\prime}\right), u \in L^{2}(\mathcal{J})$, and $y_{0} \in V$ we suppose that $y=\hat{y}+\mathcal{S} u$ belongs to $y \in H^{1}(0, T ; V)$. Suppose that, for $\ell \leq \operatorname{dim} \mathcal{V}$, the elements $\left\{\psi_{i}\right\}_{i=1}^{\ell}$ solve (4.5). Then, it follows that

$$
\lim _{\ell \rightarrow \infty}\left\|y-y^{\ell}\right\|_{W(0, T)}=0
$$

where $y^{\ell}=\hat{y}_{0}^{\ell}+\mathcal{S}^{\ell} u$ denotes the unique solution to (4.11).

Proof. By assumption, $y_{t}(t) \in V$ holds for almost all $t \in[0, T]$. As $\left\{\psi_{i}\right\}_{i \in \mathbb{N}}$ is a complete orthonormal basis in the separable Hilbert space $V$, we have

$$
y_{t}(t)=\sum_{i=1}^{\infty}\left\langle y_{t}(t), \psi_{i}\right\rangle_{V} \psi_{i} \quad \text { f.a.a. } t \in[0, T]
$$

and

$$
\int_{0}^{T} \sum_{i=1}^{\infty}\left|\left\langle y_{t}(t), \psi_{i}\right\rangle_{V}\right|^{2} \mathrm{~d} t=\int_{0}^{T}\left\|y_{t}(t)\right\|_{V}^{2} \mathrm{~d} t
$$

This follows from the Lebesgue dominated convergence theorem [24, p. 24], since $\left\|y_{t}(\cdot)\right\|_{V}^{2} \in L^{1}(0, T)$ holds. Moreover, $V$ is continuously embedded into $V^{\prime}$ (via the identification $H=H^{\prime}$ ). Thus, there exists a constant $C>0$ satisfying

$$
\begin{aligned}
\left\|y_{t}-\mathcal{P}^{\ell} y_{t}\right\|_{L^{2}\left(0, T ; V^{\prime}\right)}^{2} & \leq C \int_{0}^{T}\left\|y_{t}(t)-\mathcal{P}^{\ell} y_{t}(t)\right\|_{V}^{2} \mathrm{~d} t \\
& =C \int_{0}^{T} \sum_{i=\ell+1}^{\infty}\left|\left\langle y_{t}(t), \psi_{i}\right\rangle_{V}\right|^{2} \mathrm{~d} t .
\end{aligned}
$$

We proceed by proving that the right hand side in (4.14) tends to zero as $\ell \rightarrow \infty$ : For any $\ell \in \mathbb{N}$, define the mapping $F_{\ell}:[0, T] \rightarrow \mathbb{R}$ by

$$
F_{\ell}(t)=\sum_{i=\ell+1}^{\infty}\left|\left\langle y_{t}(t), \psi_{i}\right\rangle_{V}\right|^{2} \quad \text { f.a.a. } t \in[0, T]
$$

From (4.13) it follows that $F_{\ell} \in L^{1}(0, T)$ for all $\ell \in \mathbb{N}$. Moreover,

$$
\lim _{\ell \rightarrow \infty} F_{\ell}(t)=0 \quad \text { and } \quad\left|F_{\ell}(t)\right| \leq\left\|y_{t}(t)\right\|_{V}^{2} \quad \text { f.a.a. } t \in[0, T]
$$


and

$$
F_{\ell}(t) \leq\left\|y_{t}(t)\right\|_{V}^{2} \text { f.a.a. } t \in[0, T] \quad \text { with } \quad\left\|y_{t}(\cdot)\right\|_{V}^{2} \in L^{1}(0, T) .
$$

Now it follows from (4.14) and the Lebesgue dominated convergence theorem [24, p. 24] that

$$
\left\|y_{t}-\mathcal{P}^{\ell} y_{t}\right\|_{L^{2}\left(0, T ; V^{\prime}\right)}^{2} \rightarrow 0 \quad \text { as } \ell \rightarrow \infty .
$$

From (4.11b) we obtain $\left\langle y^{\ell}(0)-y_{0}, \psi\right\rangle_{H}=0$ for all $\psi \in V^{\ell}$. As $y_{0} \in V$ by assumption, we have $\mathcal{P}^{\ell} y_{0} \in V^{\ell}$ and

$$
y_{0}=\sum_{i=1}^{\infty}\left\langle y_{0}, \psi_{i}\right\rangle_{V} \psi_{i}
$$

Choosing $\psi=y^{\ell}(0)-\mathcal{P}^{\ell} y_{0} \in V^{\ell}$ it follows that

$$
\begin{aligned}
0 & =\left\langle y^{\ell}(0)-y_{0}, y^{\ell}(0)-\mathcal{P}^{\ell} y_{0}\right\rangle_{H}=\left\langle y^{\ell}(0)-\mathcal{P}^{\ell} y_{0}+\mathcal{P}^{\ell} y_{0}-y_{0}, y^{\ell}(0)-\mathcal{P}^{\ell} y_{0}\right\rangle_{H} \\
& =\left\|y^{\ell}(0)-\mathcal{P}^{\ell} y_{0}\right\|_{H}^{2}+\left\langle\mathcal{P}^{\ell} y_{0}-y_{0}, y^{\ell}(0)-\mathcal{P}^{\ell} y_{0}\right\rangle_{H} .
\end{aligned}
$$

Hence,

$$
\left\|y^{\ell}(0)-\mathcal{P}^{\ell} y_{0}\right\|_{H}^{2}=\left\langle y_{0}-\mathcal{P}^{\ell} y_{0}, y^{\ell}(0)-\mathcal{P}^{\ell} y_{0}\right\rangle_{H} \leq\left\|y_{0}-\mathcal{P}^{\ell} y_{0}\right\|\left\|_{H}\right\| y^{\ell}(0)-\mathcal{P}^{\ell} y_{0} \|_{H},
$$

which gives

$$
\left\|y^{\ell}(0)-\mathcal{P}^{\ell} y_{0}\right\|_{H} \leq\left\|y_{0}-\mathcal{P}^{\ell} y_{0}\right\|_{H} .
$$

Thus, using (4.16) we arrive at

$$
\begin{aligned}
0 & \leq \lim _{\ell \rightarrow \infty}\left\|y^{\ell}(0)-\mathcal{P}^{\ell} y_{0}\right\|_{H} \leq \lim _{\ell \rightarrow \infty}\left\|y_{0}-\mathcal{P}^{\ell} y_{0}\right\|_{H} \\
& =\lim _{\ell \rightarrow \infty}\left\|\sum_{i=\ell+1}^{\infty}\left\langle y_{0}, \psi_{i}\right\rangle_{H} \psi_{i}\right\|_{H}=0 .
\end{aligned}
$$

From Proposition 4.3, (4.14) and (4.18) the claim follows.

Remark 4.5. 1) Due to the continuous embedding of $W(0, T)$ into the space $C([0, T] ; H)$, Proposition 4.4 implies $y^{\ell} \rightarrow y$ in $C([0, T] ; H)$ as $\ell \rightarrow \infty$. In particular, $y^{\ell}(T)$ converges to $y(T)$ in $H$ as $\ell$ tends to $\infty$.

2) Let us mention that the convergence result in Proposition 4.4 is true for any fixed $u$ provided that the system $\left\{\psi_{i}\right\}_{i=1}^{\infty}$ computed from the snapshots associated with $u$ is complete.

4.4 POD Galerkin scheme for the adjoint equation. It is more or less clear that the convergence result $y^{\ell} \rightarrow y$ implies an associated one for the adjoint states, i.e. $p^{\ell} \rightarrow p$ as $\ell \rightarrow \infty$. This is expressed in the next result.

We turn to the POD Galerkin scheme for the adjoint system (2.6a). For that purpose let $u \in L^{2}(\mathcal{J})$ be arbitrarily given, $\left\{\psi_{1}, \ldots, \psi_{\ell}\right\}$ the associated POD basis of rank $\ell$, and let $y^{\ell} \in H^{1}\left(0, T ; V^{\ell}\right)$ denote the unique solution to (4.11). Then, $p^{\ell}=\sum_{i=1}^{\ell} p_{i}(t) \psi_{i}$ satisfies the linear system

$$
\begin{aligned}
-\frac{\mathrm{d}}{\mathrm{d} t}\left\langle p^{\ell}(t), \psi\right\rangle_{H}+a\left(p^{\ell}(t), \psi\right) & =\alpha_{1}\left\langle z_{1}-\mathcal{C} y^{\ell}, \mathcal{C} \psi\right\rangle_{W_{1}} \text { f.a.a. } t \in[0, T], \forall \psi \in V^{\ell}, \\
\left\langle p^{\ell}(T), \psi\right\rangle_{H} & =\alpha_{2}\left\langle z_{2}-\mathcal{D} y^{\ell}(T), \mathcal{D} \psi\right\rangle_{W_{2}} \quad \forall \psi \in V^{\ell} .
\end{aligned}
$$


Analogously to the arguments for the solvability of (4.11) it follows that for any $\left(z_{1}, z_{2}\right) \in W_{1} \times W_{2}$ there exists a unique solution $p^{\ell} \in H^{1}\left(0, T ; V^{\ell}\right)$ to (4.19); see [12, Proposition 3.5]. Furthermore, we find that

$$
\mathcal{B}^{\star} p^{\ell}=\mathcal{S}_{\ell}^{\star}\left(\Xi\left(z_{1}, z_{2}\right)-\Theta\left(y^{\ell}\right)\right)
$$

compare Lemma 4.3 in [12] and Lemma 2.4 above.

Proposition 4.6. For given $r \in L^{2}\left(0, T ; V^{\prime}\right), u \in L^{2}(\mathcal{J}), y_{0} \in H$ suppose that $y=\hat{y}+\mathcal{S} u$ belongs to $H^{1}(0, T ; V)$. Suppose that for $\ell \leq \operatorname{dim} \mathcal{V}$ the elements $\left\{\psi_{i}\right\}_{i=1}^{\ell}$ solve (4.5). Let $y^{\ell}=\hat{y}_{0}^{\ell}+\mathcal{S}^{\ell} u, p$, and $p^{\ell}$ be the solutions to (4.11), (2.6) and (4.19), respectively. Then there exists a constant $C>0$ depending on $\alpha_{1}, \alpha_{2}$, $\mathcal{C}$, and $\mathcal{D}$

$$
\begin{aligned}
\left\|p-p^{\ell}\right\|_{L^{2}(0, T ; V)} \leq & C\left(\left\|p(T)-\mathcal{P}^{\ell} p(T)\right\|_{H}+\left\|p-\mathcal{P}^{\ell} p\right\|_{W(0, T)}\right) \\
& +C\left(\left\|y(T)-y^{\ell}(T)\right\|_{H}+\left\|y-y^{\ell}\right\|_{L^{2}(0, T ; H)}\right) .
\end{aligned}
$$

where the linear projector $\mathcal{P}^{\ell}: V \rightarrow V^{\ell}$ is given by (4.9). If, in addition, $y_{0} \in V$ and $p \in H^{1}(0, T ; V)$ hold, then $\lim _{\ell \rightarrow \infty}\left\|p-p^{\ell}\right\|_{L^{2}(0, T ; V)}=0$ holds.

Proof. Proceeding as in the proof of Proposition 4.7 in [12] we find (4.21). Since $W(0, T)$ and $L^{2}(0, T ; H)$ are continuously embedded into $C([0, T] ; H)$ there exists a constant $C_{E}>0$ such that

$$
\left\|y(T)-y^{\ell}(T)\right\|_{H}+\left\|y-y^{\ell}\right\|_{L^{2}(0, T ; H)} \leq C_{E}\left\|y-y^{\ell}\right\|_{W(0, T)} .
$$

Thus, if $y_{0} \in V$ holds, we infer $\lim _{\ell \rightarrow 0}\left\|y-y^{\ell}\right\|_{W(0, T)}=0$ from Propositions 4.3 and 4.8. The reminder of the proof is a variant of the proof of Proposition 4.8. For the details we refer the reader to the Appendix.

Remark 4.7. Arguing as in Remark 4.5-2) we derive that the convergence result of Proposition 4.6 remains true if the POD basis is computed using an input $\tilde{u} \in L^{2}(\mathcal{J})$ that differs from $u$. Of course, the convergence rate of $p^{\ell}$ to $p$ as $\ell \rightarrow \infty$ depends on the approximation properties of the POD basis for the adjoint variable $p$; see $[7,12]$.

4.5 POD approximation of $(\hat{\mathbf{P}})$. The Galerkin projection of $(\hat{\mathbf{P}})$ leads to the discretized optimal control problem

$$
\min \hat{J}^{\ell}(u) \text { s.t. } u \in U_{\text {ad }},
$$

where $\hat{J}^{\ell}(u)=J\left(y^{\ell}(u), u\right)$ is the reduced objective function and $y^{\ell}(u)$ denotes the solution to (4.11) associated with $u \in U_{\text {ad }}$. We call $\left(\hat{\mathbf{P}}^{\ell}\right)$ a reduced-order model for $(\hat{\mathbf{P}})$.

Problem $\left(\hat{\mathbf{P}}^{\ell}\right)$ admits a unique optimal solution $\bar{u}^{\ell}$ that is interpreted as a suboptimal solution to $(\hat{\mathbf{P}})$. First-order necessary optimality conditions for $\left(\hat{\mathbf{P}}^{\ell}\right)$ are given by

$$
\left\langle\sigma \bar{u}^{\ell}-\mathcal{B}^{\star} \bar{p}^{\ell}, u-\bar{u}^{\ell}\right\rangle_{L^{2}(\mathcal{J})} \geq 0 \quad \text { for all } u \in U_{\text {ad }},
$$

where, $\bar{y}^{\ell} \in H^{1}\left(0, T ; V^{\ell}\right)$ denotes the optimal state solving (4.11) with $u=\bar{u}$ and $\bar{p}^{\ell} \in H^{1}\left(0, T ; V^{\ell}\right)$ is the adjoint state for the POD model. 
4.6 Convergence of the suboptimal controls. We proceed similarly as in $[12$, Section 4]. However, an essential difference is that we derive convergence results utilizing a POD basis of rank $\ell$ that is not necessarily related to the optimal control $\bar{u}$ as an input function for the generation of the snapshots.

Proposition 4.8. Suppose that the POD basis of rank $\ell$ is computed using an arbitrarily chosen $u \in L^{2}(\mathcal{J})$. Let $\bar{u}$ and $\bar{u}^{\ell}$ be the optimal solutions to $(\hat{\mathbf{P}})$ and $\left(\hat{\mathbf{P}}^{\ell}\right)$, respectively. Moreover, $\bar{p} \in W(0, T)$ denotes the adjoint state associated with $\bar{u}$. Then,

where $\hat{p}^{\ell}$ solves

$$
\left\|\bar{u}-\bar{u}^{\ell}\right\|_{L^{2}(\mathcal{J})} \leq c\left\|\bar{p}-\hat{p}^{\ell}\right\|_{L^{2}(0, T ; V)},
$$

$-\frac{\mathrm{d}}{\mathrm{d} t}\left\langle\hat{p}^{\ell}(t), \psi\right\rangle_{H}+a\left(\hat{p}^{\ell}(t), \psi\right)=\alpha_{1}\left\langle z_{1}-\mathcal{C} \hat{y}^{\ell}, \mathcal{C} \psi\right\rangle_{W_{1}}$ f.a.a. $t \in[0, T], \forall \psi \in V^{\ell}$,

$$
\left\langle\hat{p}^{\ell}(T), \psi\right\rangle_{H}=\alpha_{2}\left\langle z_{2}-\mathcal{D} \hat{y}^{\ell}(T), \mathcal{D} \psi\right\rangle_{W_{2}} \quad \forall \psi \in V^{\ell}
$$

and $\hat{y}^{\ell}$ is the solution to

$$
\begin{gathered}
\frac{\mathrm{d}}{\mathrm{d} t}\left\langle\hat{y}^{\ell}(t), \psi\right\rangle_{H}+a\left(\hat{y}^{\ell}(t), \psi\right)=\langle(r+\mathcal{B} \bar{u})(t), \psi\rangle_{V^{\prime}, V} \text { f.a.a. } t \in[0, T], \forall \psi \in V^{\ell}, \\
\left\langle\hat{y}^{\ell}(0), \psi\right\rangle_{H}=\left\langle y_{0}, \psi\right\rangle_{H} \quad \forall \psi \in V^{\ell} .
\end{gathered}
$$

Proof. The proof is a variant of the proof of Theorem 4.5 in [12]. For more details we refer to the Appendix.

Notice that $\hat{p}^{\ell}$ is the POD-approximate associated with $\hat{y}^{\ell}$ and $\hat{y}^{\ell}=\hat{y}_{0}^{\ell}+\mathcal{S}^{\ell} \bar{u}$. Therefore, both $\hat{y}^{\ell}$ and $\hat{p}^{\ell}$ are associated with the same optimal control $\bar{u}$ so that we can apply Proposition 4.3 and Proposition 4.6 to estimate the difference $\bar{y}-\hat{y}^{\ell}$ and $\bar{p}-\hat{p}^{\ell}$, respectively. In contrast to this, $\bar{y}^{\ell}=\hat{y}_{0}^{\ell}+\mathcal{S}^{\ell} \bar{u}^{\ell}$ corresponds to the suboptimal control $\bar{u}^{\ell}$, which we estimate in the next theorem.

Theorem 4.9. Suppose that the POD basis of rank $\ell$ is computed using an arbitrarily chosen $u \in L^{2}(\mathcal{J})$. Let $\bar{u}$ and $\bar{u}^{\ell}$ be the optimal solutions to $(\hat{\mathbf{P}})$ and $\left(\hat{\mathbf{P}}^{\ell}\right)$, respectively. Moreover, let $\bar{y}$ and $\bar{p}$ denote the optimal state and adjoint, respectively, associated with $\bar{u}$. Then there exists a constant $C>0$ not depending on $\ell$ such that

$$
\begin{aligned}
& \left\|\bar{u}-\bar{u}^{\ell}\right\|_{L^{2}(\mathcal{J})} \\
& \quad \leq C\left(\left\|\bar{y}-\mathcal{P}^{\ell} \bar{y}\right\|_{W(0, T)}+\left\|\bar{y}^{\ell}(0)-\mathcal{P}^{\ell} y_{0}\right\|_{H}+\left\|\bar{p}-\mathcal{P}^{\ell} \bar{p}\right\|_{W(0, T)}\right),
\end{aligned}
$$

where the linear projector $\mathcal{P}^{\ell}: V \rightarrow V^{\ell}$ is given in (4.9).

If, in addition, $y_{\circ} \in V$ and $\bar{y}, \bar{p} \in H^{1}(0, T ; V)$ hold and $\left\{\psi_{i}\right\}_{i=1}^{\infty}$ is a complete orthonormal basis for $V$, then

$$
\lim _{\ell \rightarrow \infty}\left\|\bar{u}-\bar{u}^{\ell}\right\|_{L^{2}(\mathcal{J})}=0 .
$$

Proof. Combining (4.21) and (4.23) we find

$$
\begin{aligned}
\left\|\bar{u}-\bar{u}^{\ell}\right\|_{L^{2}(\mathcal{J})} \leq & c C\left(\left\|\bar{p}(T)-\mathcal{P}^{\ell} \bar{p}(T)\right\|_{H}+\left\|\bar{p}-\mathcal{P}^{\ell} \bar{p}\right\|_{W(0, T)}\right) \\
& +C\left(\left\|\bar{y}(T)-y^{\ell}(T)\right\|_{H}+\left\|\bar{y}-y^{\ell}\right\|_{L^{2}(0, T ; H)}\right) .
\end{aligned}
$$


Since $W(0, T)$ is contiunuously embedded into $C([0, T] ; H)$ we derive

$$
\left\|\bar{u}-\bar{u}^{\ell}\right\|_{L^{2}(\mathcal{J})} \leq \tilde{C}\left(\left\|\bar{p}-\mathcal{P}^{\ell} \bar{p}\right\|_{W(0, T)}+\left\|\bar{y}-y^{\ell}\right\|_{W(0, T)}\right)
$$

for a constant $\tilde{C}>0$. Now the claim follows from Propositions 4.3, 4.4, and 4.6.

Remark 4.10. Let us consider the following idealized situation [12]: Let $\bar{u}$ be the optimal solution to $(\hat{\mathbf{P}})$. Moreover, let $\bar{y}, \bar{p} \in H^{1}(0, T ; V)$ denote the optimal state and adjoint state, respectively, associated with $\bar{u}$ and let $y_{0} \in V$. Then we consider the minimization problem

$$
\min _{\psi_{1}, \ldots, \psi_{\ell}}\left\|\bar{y}-\mathcal{P}^{\ell} \bar{y}\right\|_{H^{1}(0, T ; V)}^{2}+\left\|\bar{p}-\mathcal{P}^{\ell} \bar{p}\right\|_{H^{1}(0, T ; V)}^{2} \text { s.t. }\left\langle\psi_{i}, \psi_{j}\right\rangle_{V}=\delta_{i j}, 1 \leq i, j \leq \ell .
$$

Its solution $\left\{\bar{\psi}_{i}\right\}_{i=1}^{\ell}$ of rank $\ell$ satisfies the eigenvalue problem

$$
\overline{\mathcal{R}} \bar{\psi}_{i}=\bar{\lambda}_{i} \bar{\psi}_{i}, \quad 1 \leq i \leq \ell,
$$

where the linear, bounded, non-negative and self-adjoint operator $\overline{\mathcal{R}}$ is defined as

$$
\overline{\mathcal{R}} z=\int_{0}^{T}\langle\bar{y}(t), z\rangle_{V} y(t)+\left\langle\bar{y}_{t}(t), z\right\rangle_{V} \bar{y}_{t}(t)+\langle\bar{p}(t), z\rangle_{V} \bar{p}(t)+\left\langle\bar{p}_{t}(t), z\right\rangle_{V} \bar{p}_{t}(t) \mathrm{d} t
$$

for $z \in V$. Then, (4.26) can be replaced by

$$
\left\|\bar{u}-\bar{u}^{\ell}\right\|_{L^{2}(\mathcal{J})}^{2} \leq \bar{C}\left(\left\|\bar{y}^{\ell}(0)-\mathcal{P}^{\ell} y_{0}\right\|_{H}^{2}+\sum_{i=\ell+1}^{\infty} \bar{\lambda}_{i}\right)
$$

with a constant $\bar{C}>0$. Now we can estimate the decay of the norms $\left\|\bar{y}-\mathcal{P}^{\ell} \bar{y}\right\|_{W(0, T)}$ and $\left\|\bar{p}-\mathcal{P}^{\ell} \bar{p}\right\|_{W(0, T)}$ in (4.26) in terms of the eigenvalues $\bar{\lambda}_{i}$ and obtain an error estimate with respect to the remainder $\sum_{i=\ell+1}^{\infty} \bar{\lambda}_{i}$. In contrast to this, the decay of the eigenvalues $\lambda_{i}$ can only be used to bound $\left\|\bar{y}-\mathcal{P}^{\ell} \bar{y}\right\|_{L^{2}(0, T ; V)}$ from above, but not the expression $\left\|\bar{y}_{t}-\mathcal{P}^{\ell} \bar{y}_{t}\right\|_{L^{2}\left(0, T ; V^{\prime}\right)}+\left\|\bar{p}-\mathcal{P}^{\ell} \bar{p}\right\|_{W(0, T)}$.

4.7 A-posteriori error estimate for the POD approximation. In this subsection, we complete the discussion of the a-posteriori estimate by combining Theorem 4.9 and Proposition 3.2. The proposition permits to estimate $\left\|\bar{u}-\bar{u}^{\ell}\right\|$ by the norm of an appropriate $\zeta$, while Theorem 4.9 will be used to show that $\zeta$ tends to zero as $\ell \rightarrow \infty$, since it ensures convergence of $\bar{u}^{\ell}$ to the optimal solution $\bar{u}$ of $(\hat{\mathbf{P}})$.

For any $\ell$ let $\bar{u}^{\ell} \in U_{\text {ad }}$ be the optimal solution to $\left(\hat{\mathbf{P}}^{\ell}\right)$. This optimal $\bar{u}^{\ell}$ is taken as a suboptimal $u_{p}$ for $(\hat{\mathbf{P}})$, i.e. in Proposition 3.2 we take $u_{p}:=\bar{u}^{\ell}$.

Theorem 4.11. 1) Let $\ell \leq d$ be arbitrarily given and $\bar{u}^{\ell} \in U_{\text {ad }}$ be the optimal solution to $\left(\hat{\mathbf{P}}^{\ell}\right)$. Denote by $\tilde{y}=\tilde{y}\left(\bar{u}^{\ell}\right)=\hat{y}_{0}+\mathcal{S} \bar{u}^{\ell}$ the solution to (2.2) with $u=\bar{u}^{\ell}$ and let $\tilde{p}=\tilde{p}\left(\bar{u}^{\ell}\right)$ solve the associated adjoint equation

$$
\begin{aligned}
-\frac{\mathrm{d}}{\mathrm{d} t}\langle\tilde{p}(t), \varphi\rangle_{H}+a(\tilde{p}(t), \varphi) & =\alpha_{1}\left\langle z_{1}-\mathcal{C} \tilde{y}, \mathcal{C} \varphi\right\rangle_{W_{1}} \text { f.a.a. } t \in[0, T], \forall \varphi \in V, \\
\langle\tilde{p}(T), \varphi\rangle_{H} & =\alpha_{2}\left\langle z_{2}-\mathcal{D} \tilde{y}(T), \mathcal{D} \varphi\right\rangle_{W_{2}} \quad \forall \varphi \in V .
\end{aligned}
$$

Define, according to (3.5), the function $\zeta^{\ell} \in L^{2}(\mathcal{J})$ by

$$
\zeta^{\ell}(s)= \begin{cases}{\left[\left(\sigma \bar{u}^{\ell}-\mathcal{B}^{\star} \tilde{p}\left(\bar{u}^{\ell}\right)\right)(s)\right]_{-}} & \text {on } \mathcal{A}_{-}^{\ell}=\left\{s \in \mathcal{J} \mid \bar{u}^{\ell}(s)=u_{a}(s)\right\}, \\ {\left[\left(\sigma \bar{u}^{\ell}-\mathcal{B}^{\star} \tilde{p}\left(\bar{u}^{\ell}\right)\right)(s)\right]_{+}} & \text {on } \mathcal{A}_{+}^{\ell}=\left\{s \in \mathcal{J} \mid \bar{u}^{\ell}(s)=u_{b}(s)\right\}, \\ -\left(\sigma \bar{u}^{\ell}-\mathcal{B}^{\star} \tilde{p}^{\ell}\left(\bar{u}^{\ell}\right)\right)(s) & \text { on } \mathcal{J}^{\ell}=\mathcal{J} \backslash\left(\mathcal{A}_{-}^{\ell} \cup \mathcal{A}_{+}^{\ell}\right) .\end{cases}
$$


Then

$$
\left\|\bar{u}-\bar{u}^{\ell}\right\|_{L^{2}(\mathcal{J})} \leq \frac{1}{\sigma}\left\|\zeta^{\ell}\right\|_{L^{2}(\mathcal{J})} .
$$

2) If all hypotheses of Proposition 4.6 and Theorem 4.9 are satisfied, in particular $\left\{\psi_{i}\right\}_{i=1}^{\infty}$ is a complete orthonormal basis for $V$, then the sequences $\left\{\bar{u}^{\ell}\right\}_{\ell \in \mathbb{N}}$ and $\left\{\mathcal{B}^{\star} \hat{p}^{\ell}\right\}_{\ell \in \mathbb{N}}$ converge to $\bar{u}$ respectively $\mathcal{B}^{\star} \bar{p}$ in $L^{2}(\mathcal{J})$ as $\ell \rightarrow \infty$ and

$$
\left\|\zeta^{\ell}\right\|_{L^{2}(\mathcal{J})} \rightarrow 0
$$

Proof. By Theorem 4.9, the sequences $\left\{\bar{u}^{\ell}\right\}_{\ell \in \mathbb{N}}$ and $\left\{\mathcal{B}^{\star} \bar{p}^{\ell}\right\}_{\ell \in \mathbb{N}}$ converge to $\bar{u}$ respectively $\mathcal{B}^{\star} \bar{p}$ in $L^{2}(\mathcal{J})$. There exist subsequences $\left\{\bar{u}^{\ell_{k}}\right\}_{k \in \mathbb{N}}$ and $\left\{\mathcal{B}^{\star} \bar{p}^{\ell_{k}}\right\}_{k \in \mathbb{N}}$ satisfying

$$
\lim _{k \rightarrow \infty} \bar{u}^{\ell_{k}}(s)=\bar{u}(s) \quad \text { and } \quad \lim _{k \rightarrow \infty} \mathcal{B}^{\star} \bar{p}^{\ell_{k}}(s)=\mathcal{B}^{\star} \bar{p}(s) \quad \text { f.a.a. } s \in \mathcal{J} \text {. }
$$

Next we consider the active and inactive sets for $\bar{u}$.

1) Let $s \in \mathcal{J}=\left\{s \in \mathcal{J} \mid u_{a}(s)<\bar{u}(s)<u_{b}(s)\right\}$. For $k_{\circ}=k_{\circ}(s) \in \mathbb{N}$ sufficiently large, $\bar{u}^{\ell_{k}}(s) \in\left(u_{a}(s), u_{b}(s)\right)$ for all $k \geq k_{\circ}$ and f.a.a. $s \in \mathcal{J}$. Thus, $\sigma \bar{u}^{\ell_{k}}(s)-$ $\mathcal{B}^{\star} \bar{p}^{\ell_{k}}(s)=0$ for all $k \geq k_{\circ}(s)$ in $\mathcal{J}$ a.e. This implies

$$
\zeta^{\ell_{k}}(s)=0 \quad \forall k \geq k_{\circ}(s) \text { and f.a.a. } s \in \mathcal{J}
$$

2) Suppose that $s \in \mathcal{A}_{-}=\left\{s \in \mathcal{J} \mid u_{a}(s)=\bar{u}(s)\right\}$. From $\sigma \bar{u}(s)-\mathcal{B}^{\star} \bar{p}(s) \geq 0$ in $\mathcal{A}_{-}$a.e. we deduce

$$
\lim _{k \rightarrow \infty} \zeta^{\ell_{k}}(s)=\left[\left(\sigma \bar{u}^{\ell}-\mathcal{B}^{\star} \bar{p}^{\ell}\right)(s)\right]_{-}=0 \quad \text { f.a.a. } s \in \mathcal{A}_{-} .
$$

3) Suppose that $s \in \mathcal{A}_{+}=\left\{s \in \mathcal{J} \mid u_{b}(s)=\bar{u}(s)\right\}$. Analogously to part 2) we find

$$
\lim _{k \rightarrow \infty} \zeta^{\ell_{k}}(s)=\left[\left(\sigma \bar{u}^{\ell}-\mathcal{B}^{\star} \bar{p}^{\ell}\right)(s)\right]_{+}=0 \quad \text { f.a.a. } s \in \mathcal{A}_{-} .
$$

Combining (4.29)-(4.31) we conclude that $\lim _{k \rightarrow \infty} \zeta^{\ell_{k}}=0$ a.e. in $(0, T)$. Utilizing the dominated convergence theorem $[24$, p. 24] we have

$$
\lim _{k \rightarrow \infty}\left\|\zeta^{\ell_{k}}\right\|_{L^{2}(\mathcal{J})}=0 \text {. }
$$

Since all subsequences contain a subsequence converging to zero, the claim follows from a standard argument.

Remark 4.12. $\quad 1$ ) Notice that $\tilde{y}$ and $\tilde{p}$ must be taken as the solutions to the (full) state and adjoint equation, respectively, not of their POD-approximations.

2) Part 2) of Theorem 4.11 shows that $\left\|\zeta^{\ell}\right\|_{L^{2}(\mathcal{J})}$ can be expected smaller than any $\varepsilon>0$ provided that $\ell$ is taken sufficiently large. Motivated by this result, we set up the Algorithm 1.

Remark 4.13. In the numerical realization of Algorithm 1, Step 6 requires the solution of the state as well as of the adjoint equation by, e.g., a finite element or finite differerence scheme. In Section 5, Run 1, we will see that the main part of the CPU time for Algorithm 1 is consumed by step 6 . 


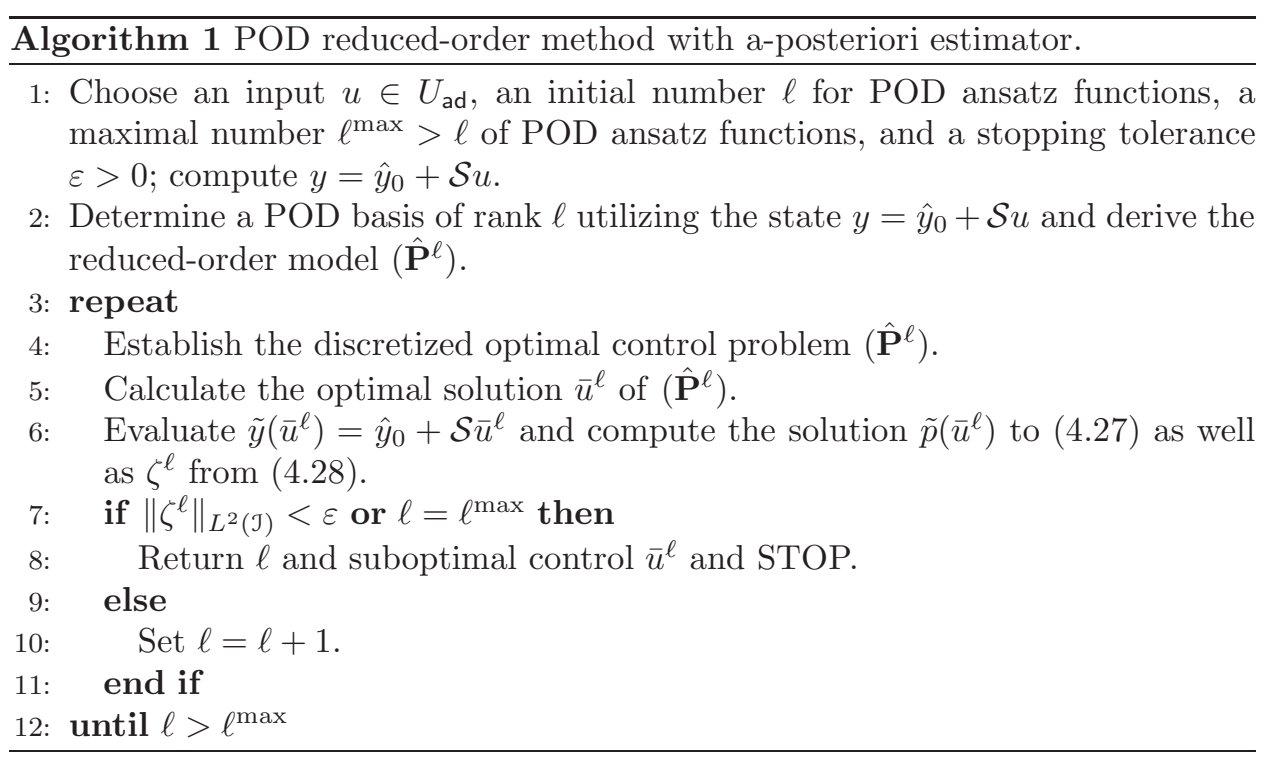

\section{Numerical experiments}

In this section we present two numerical test examples, where the first one is a parabolic problem as in Example 2.3, while the second one is of elliptic type and demonstrates that our method applies also to other types of problems (see Remark 3.3-2). All coding is done in MATLAB using routines from the FEMLAB 2.2 package for the finite element (FE) implementation.

Run 1 (Parabolic example). Let $\Omega=(0,1) \times(0,1)$ be the open unit square, $T=1$ and $Q=(0, T) \times \Omega$. We set

$$
\Gamma_{N}=\left\{\left(x_{1}, x_{2}\right) \mid x_{1} \in\{0,1\} \text { and } x_{2} \in[0,1]\right\} \quad \text { and } \quad \Gamma_{C}=\Gamma \backslash \Gamma_{N} .
$$

We consider the minimization problem

$$
\min \frac{1}{2} \int_{\Omega}(y(T, \mathbf{x})-20)^{2} \mathrm{~d} \mathbf{x}+\frac{1}{400} \int_{0}^{T} u(t)^{2} \mathrm{~d} t
$$

subject to the heat equation

$$
\begin{aligned}
y_{t}(t, \mathbf{x})-\Delta y(t, \mathbf{x}) & =0 & & \text { f.a.a. }(t, \mathbf{x}) \in Q, \\
\frac{\partial y}{\partial n}(t, \mathbf{x}) & =0 & & \text { f.a.a. }(t, \mathbf{x}) \in \Sigma_{N}=(0, T) \times \Gamma_{N}, \\
\frac{\partial y}{\partial n}(t, \mathbf{x}) & =u(t) & & \text { f.a.a. }(t, \mathbf{x}) \in \Sigma_{C}=(0, T) \times \Gamma_{C}, \\
y(0, \mathbf{x}) & =30 & & \text { f.a.a. } \mathbf{x} \in \Omega
\end{aligned}
$$

and to the bilateral control constraints

$$
-6 \leq u(t) \leq 1 \quad \text { f.a.a. } t \in(0, T) .
$$

We discretize the domain $\Omega$ by a uniform rectangular triangulation with mesh-size $h=1 / 39$; see Figure 1 . For the time integration we apply an implicit Euler method with step size $\tau=1 / 300$. Since we do not know the exact optimal control $\bar{u}$, we take the FE solution $\bar{u}^{h, \tau}$ to $(\hat{\mathbf{P}})$ as a substitute, considering the mesh with $h=1 / 39$ 

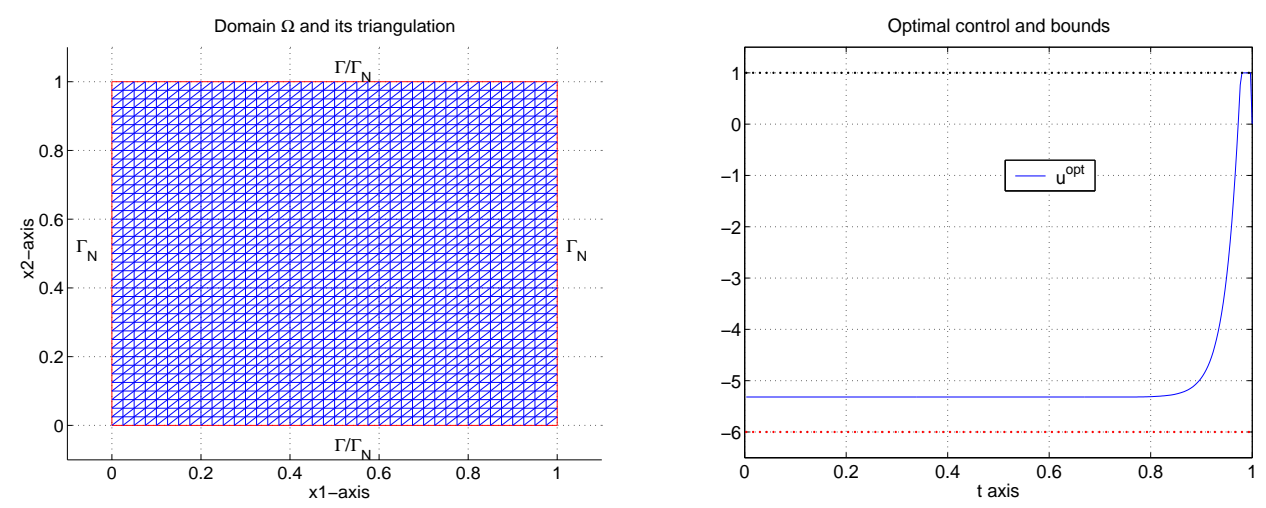

Figure 1. Run 1: Domain $\Omega$ (left plot) and optimal FE control with lower and upper bound(right plot).

as sufficiently fine; see Figure 1. The reduced optimal control problem $(\hat{\mathbf{P}})$ as well as its low-order approximation $\left(\hat{\mathbf{P}}^{\ell}\right)$ are solved by a primal-dual active set strategy, cf. [11], where the linear systems in each level of the algorithm are treated by the preconditioned conjugate gradient method. According to Theorem 4.11, we compare the error $\bar{u}^{h, \tau}-\bar{u}^{\ell}$ with the norm of $\zeta^{\ell}$ for different values of $\ell$ in Table 1 . It turns out that the norms decay with increasing $\ell$. Moreover, $\sigma^{-1}\left\|\zeta^{\ell}\right\|_{L^{2}(0, T)}$ is

\begin{tabular}{cc|c}
\hline$\ell$ & $\left\|\bar{u}^{h, \tau}-\bar{u}^{\ell}\right\|_{L^{2}(0, T)}$ & $\frac{1}{\sigma}\left\|\zeta^{\ell}\right\|_{L^{2}(0, T)}$ \\
\hline 2 & 0.079670 & 3.361904 \\
3 & 0.016831 & 0.065327 \\
4 & 0.001876 & 0.002951 \\
5 & 0.000943 & 0.002229 \\
\hline
\end{tabular}

\begin{tabular}{lr}
\hline Computation part & CPU time \\
\hline FE optimizer & $1611 \mathrm{~s}$ \\
Step 1 & $8 \mathrm{~s}$ \\
Step 2 with $\ell^{\max }=10$ & $5 \mathrm{~s}$ \\
Step 4 for $\ell=6$ & $\ll 1 \mathrm{~s}$ \\
Step 5 for $\ell=6$ & $3 \mathrm{~s}$ \\
Step 6 for $\ell=6$ & $18 \mathrm{~s}$ \\
\hline
\end{tabular}

TABLE 1. Run 1: Norms $\left\|\bar{u}^{h, \tau}-\bar{u}^{\ell}\right\|_{L^{2}(0, T)}$ and $\sigma^{-1}\left\|\zeta^{\ell}\right\|_{L^{2}(0, T)}$ for different $\ell$ (left table); CPU times for different parts needed to carry out Algorithm 1.

an upper bound for $\left\|\bar{u}^{h, \tau}-\bar{u}^{\ell}\right\|_{L^{2}(0, T)}$ as stated in Theorem 3.1. For the CPU times refer to Table 1 . Note that for $h=1 / 39$ and $\tau=1 / 300$ we expect that $\left\|\bar{u}^{h, \tau}-\bar{u}\right\|_{L^{2}(0, T)} \sim c \cdot 9 \mathrm{e}-5$. Starting Algorithm 1 with $u=1 \in U_{\mathrm{ad}}, \ell=2$, $\ell^{\max }=10$, and choosing the tolerance $\varepsilon=10^{-2}$ the method stops after 50 seconds - compared to 1611 seconds needed for the FE optimization solver.

Run 2 (Elliptic example). In this numerical example we consider a problem motivated by acoustic applications in vehicle simulations [9, 10, 28]. Furthermore, this example is constructed in such a way that the exact optimal control is known. Suppose that the interior of the car is simplified by the two-dimensional domain $\Omega$ plotted in Figure 2. The boundary $\Gamma=\partial \Omega$ is split into two measurable disjunct 

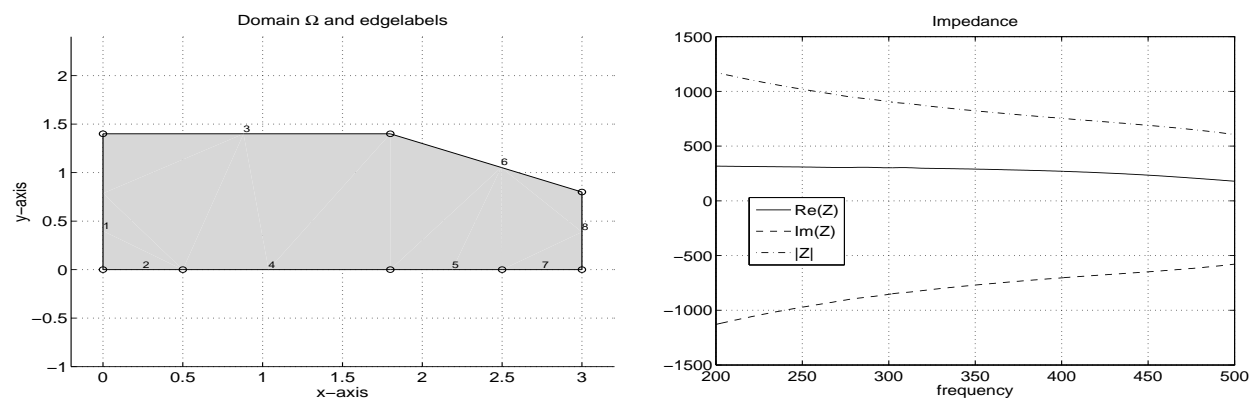

FiguRE 2. Run 2: Interior $\Omega$ of the vehicle, where the boundary part $\Gamma_{R}$ consists in parts 4 and 5 (left plot); impedance values $Z=Z_{\Re}+\jmath Z_{\Im}$ for Melamin with $50 \mathrm{~mm}$ width in the frequency range from 200 to $400 \mathrm{~Hz}$ (right plot).

parts $\Gamma_{R}$ and $\Gamma_{N}$. For given complex impedance $Z \neq 0$ (see Figure 2) the associated sound pressure $p: \Omega \rightarrow \mathbb{C}$ is governed by the Helmholtz equation

$$
\Delta p(\mathbf{x})+k^{2} p(\mathbf{x})=u b(\mathbf{x}) \quad \text { for all } \mathbf{x}=(x, y) \in \Omega,
$$

together with the boundary conditions

$$
\begin{array}{ll}
\frac{\jmath}{\varrho_{\circ} \omega} \frac{\partial p(\mathbf{x})}{\partial n}=\frac{p(\mathbf{x})}{Z} & \text { for all } \mathbf{x} \in \Gamma_{R}, \\
\frac{\jmath}{\varrho_{\circ} \omega} \frac{\partial p(\mathbf{x})}{\partial n}=0 & \text { for all } \mathbf{x} \in \Gamma_{N} .
\end{array}
$$

In (5.1a) the constant $c=343.799\left[\frac{\mathrm{m}}{\mathrm{s}}\right]$ denotes the sound of speed, $\varrho_{\circ}=1.19985\left[\frac{\mathrm{kg}}{\mathrm{m}^{3}}\right]$ is an ambient density, $f$ stands for the frequency, $\omega=2 \pi f$ is the circle frequency and $k=\omega / c$ is the wave number. The right-hand side is a simplified model for a source at $\mathbf{x}_{q}=(0.21,1.28)$ (e.g., a loudspeaker) with the intensity $|u|, u \in \mathbb{C}$, and shape function

$$
b(\mathbf{x})=\frac{1}{10} \exp \left(-\frac{1}{0.02}\left((x-0.21)^{2}+(y-1.28)^{2}\right)\right) \quad \text { for } \mathbf{x}=(x, y) \in \Omega .
$$

For the normal impedance boundary condition (5.1b) let $\jmath$ be the imaginary unit and $\frac{\partial}{\partial n}$ denote the derivative in the outward normal direction. All other parts on the boundary are assumed to be perfectly rigid, see (5.1c). We suppose that for all values of $Z \in \mathbb{C}$, plotted in Figure 2, and for all $f$ in the frequency range from 200 to $400[H z]$, problem (5.1) admits a unique solution. Due to the Fredholm theory, [24], we can ensure existence of a solution provided $k^{2}$ is not an eigenvalue of $-\Delta$ considered on $\Omega$ with Neumann and Robin boundary conditions on $\Gamma_{N}$ respectively $\Gamma_{R}$.

Now we define the data such that the optimal solution is known in advance. To this aim, let $u_{\circ}(f)=2 \cos (\pi(f-200) / 50)+2 \jmath \sin (\pi(f-200) / 50)$ (see Figure 3$)$ and let $p_{\circ}=p_{\circ}(f)$ be the unique solution to (5.1) for the choice $u=u_{\circ}(f)$ in (5.1a). We set $p_{i}^{\mathrm{m}}=p_{\circ}\left(\mathbf{x}_{i}\right), i=1, \ldots, 10$, with 10 different observation points $\mathbf{x}_{i} \in \Omega \cup \Gamma_{N}$, 

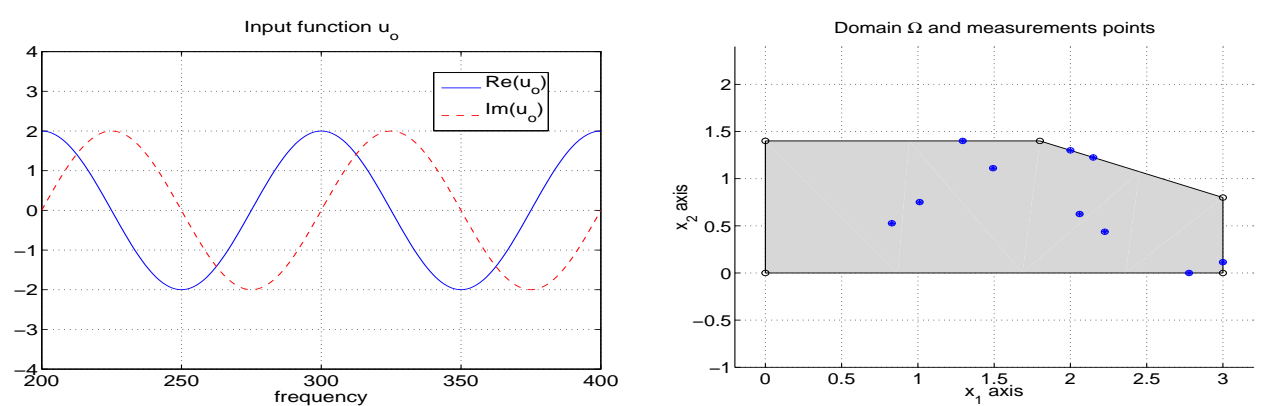

FiguRE 3. Run 2: Input function $u_{\circ}$ for the frequencies from 200 to $400 \mathrm{~Hz}$ (left plot); observation points in $\Omega \cup \Gamma_{N}$ (right plot).

$1 \leq i \leq 10$; see Figure 3. Introducing the quadratic cost functional

$$
J(p, u)=\frac{1}{200} \sum_{i=1}^{10}\left|p\left(\mathbf{x}_{i}\right)-p_{i}^{\mathrm{m}}\right|^{2}+\frac{1}{2}\left|u-u_{\circ}\right|^{2},
$$

where $u_{n}=u_{\circ}$, and $|u|$ is the absolute modulus of the convex number $u$, we consider the optimal control problem

$$
\min J(p, u) \quad \text { subject to }(p, u) \text { solves }(5.1)
$$

over the frequency range from 200 to $400[\mathrm{~Hz}]$. Notice that $\left(p_{\circ}, u_{\circ}\right)$ must be the optimal solution to (5.3). The optimality conditions to (5.3) consist of the state equation, the adjoint system

$$
\begin{aligned}
\Delta \lambda_{\circ}(\mathbf{x})+k^{2} \lambda_{\circ}(\mathbf{x}) & =\frac{1}{100} \sum_{i=1}^{10}\left(p_{i}^{\mathrm{m}}-p_{\circ}\left(\mathbf{x}_{i}\right)\right) \delta_{\mathbf{x}_{i}}(\mathbf{x}), & & \text { for all } \mathbf{x} \in \Omega, \\
\frac{\jmath}{\varrho_{\circ} \omega} \frac{\partial \lambda_{\circ}(\mathbf{x})}{\partial n} & =-\frac{\lambda_{\circ}(\mathbf{x})}{\bar{Z}} & & \text { for all } \mathbf{x} \in \Gamma_{R}, \\
\frac{\jmath}{\varrho_{\circ} \omega} \frac{\partial \lambda_{\circ}(\mathbf{x})}{\partial n} & =0 & & \text { for all } \mathbf{x} \in \Gamma_{N}
\end{aligned}
$$

and the equation

$$
\left(u_{\circ}-u_{n}\right)-\int_{\Omega} b(\mathbf{x}) \lambda_{\circ}(\mathbf{x}) \mathrm{d} \mathbf{x}=0 \quad \text { in } \mathbb{C},
$$

where $\delta_{\mathbf{x}_{i}}$ denotes the Dirac delta distribution satisfying

$$
\left\langle\delta_{\mathbf{x}_{i}}, \varphi\right\rangle=\varphi\left(\mathbf{x}_{i}\right) \quad \text { for } \varphi \in C\left(\Omega \cup \Gamma_{N}\right) \text { and } i=1, \ldots, 10 .
$$

Remark 5.14. The functional $J$ contains point observations, hence the problem - besides the fact that the state equation is of different type than in the sections before - does formally not fit in our theory. Nevertheless, the perturbation analysis can be extended, and the numerical results show the efficiency of our approach. $\diamond$

The domain $\Omega$ is discretized utilizing a standard piecewise linear FE discretization with $m=2108$ degrees of freedom. To generate the snapshot ensemble we compute the FE solution $p_{h}^{j}$ to $(5.1)$ for the frequencies $f=200,201, \ldots, 400$ and for $u=1, \jmath$. Thus, we have $n=402$ snapshots. Recall that also $\omega, k$, and $Z$ depend on $f$. In the context of Section 4.2 we choose the real part $y_{j}=\Re e\left(p_{h}^{j}\right) \in H^{1}(\Omega)$ 

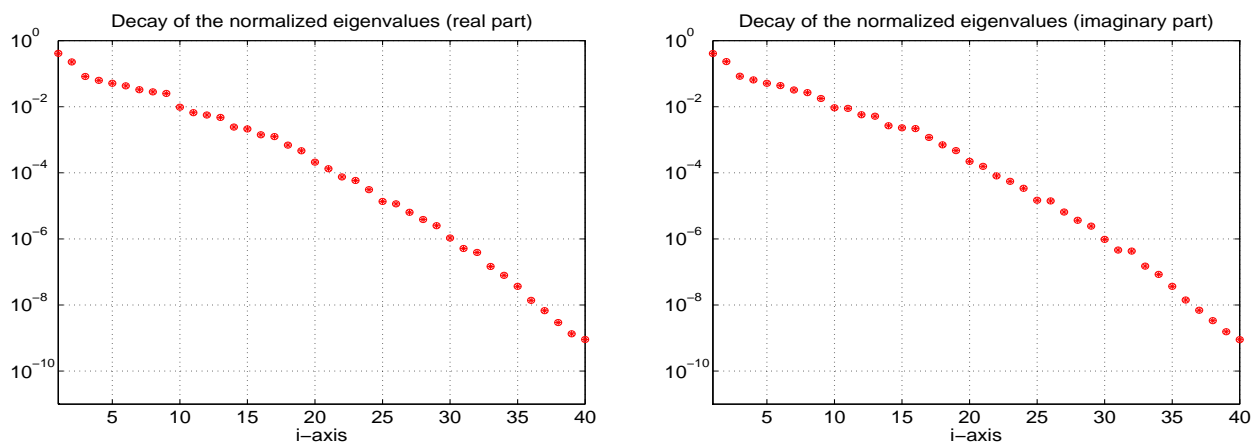

FiguRE 4. Run 2: Decay of the largest 40 normalized eigenvalues $\lambda_{i} / \sum_{i=1}^{d} \lambda_{i}$ for the real and the imaginary part of the snapshots.

of $p_{h}^{j}, 1 \leq j \leq n$, and compute the solution to the eigenvalue problem 4.8. For the decay of the largest 40 eigenvalues we refer to Figure 4. Setting

$$
\mathcal{E}_{\Re e}(\ell)=\frac{\sum_{i=1}^{\ell} \lambda_{i}}{\sum_{i=1}^{d} \lambda_{i}} \text { (real part) and } \mathcal{E}_{\Im m}(\ell)=\frac{\sum_{i=1}^{\ell} \lambda_{i}}{\sum_{i=1}^{d} \lambda_{i}} \text { (imaginary part) }
$$

we found that $\mathcal{E}_{\Re e}(\ell)$ and $\mathcal{E}_{\Im m}(\ell)$ are approximately $1-4 \cdot 10^{-10}$, i.e., very close to one. Hence, we determine a POD basis of rank 40. By $\left\{\psi_{i}\right\}_{i=1}^{\ell}$ we denote the POD basis of rank $\ell$ for the real part. For the imaginary part $\Im m\left(p_{h}^{j}\right)$ of $p_{h}^{j}$ we proceed analogously. The obtained POD basis is denoted by $\left\{\phi_{i}\right\}_{i=1}^{\ell}$ and the largest $\ell_{\max }=40$ eigenvalues are shown in the right plot of Figure 4 . For simplicity of the representation, we choose the same number of POD ansatz functions for the real and the imaginary parts which is not necessary. Now we make the POD Galerkin ansatz

$$
p^{\ell}(\mathbf{x} ; f)=\sum_{i=1}^{\ell} a_{i} \psi_{i}+\jmath b_{i} \phi_{i}, \quad a_{i}, b_{i} \in \mathbb{R}, \mathbf{x} \in \Omega, 200[H z] \leq f \leq 400[H z]
$$

with $3 \leq \ell \leq \ell_{\max }$ and derive the reduced-order model for (5.3). Then, we apply Algorithm 2. In Figure 5 the change of the number $\ell$ of POD basis functions depending on the frequencies is plotted.

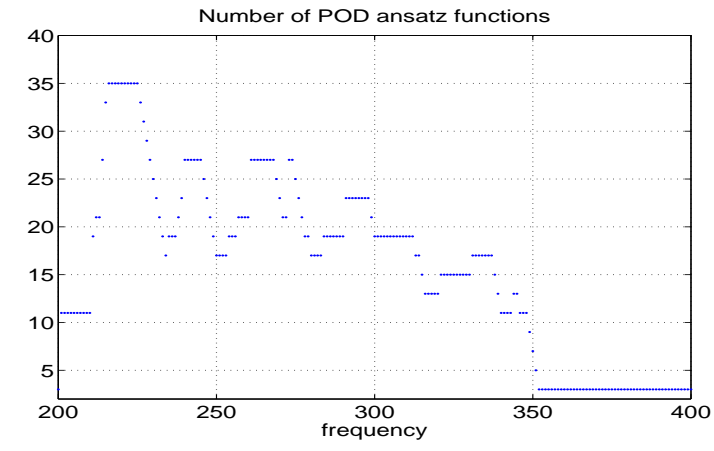

Figure 5: Change of the number $\ell$ of POD basis functions.

\begin{tabular}{cc|c}
\hline$\ell$ & $\left|\bar{u}^{h, \tau}-\bar{u}^{\ell}\right|_{\mathbb{C}}$ & $\frac{1}{\sigma}\left|\zeta^{\ell}\right|_{\mathbb{C}}$ \\
\hline 15 & $1.765 \mathrm{e}-1$ & $1.770 \mathrm{e}-1$ \\
20 & $1.709 \mathrm{e}-2$ & $1.714 \mathrm{e}-2$ \\
25 & $2.054 \mathrm{e}-3$ & $2.060 \mathrm{e}-3$ \\
30 & $8.634 \mathrm{e}-5$ & $8.660 \mathrm{e}-5$ \\
35 & $1.751 \mathrm{e}-6$ & $1.756 \mathrm{e}-6$ \\
40 & $8.555 \mathrm{e}-8$ & $8.581 \mathrm{e}-8$ \\
\hline
\end{tabular}

TABLE 3: $\left|\bar{u}-\bar{u}^{\ell}\right|_{\mathbb{C}}$ and $\frac{1}{\sigma}\left|\zeta^{\ell}\right|_{\mathbb{C}}$ for different $\ell$ and fixed frequency $f=400[H z]$. 


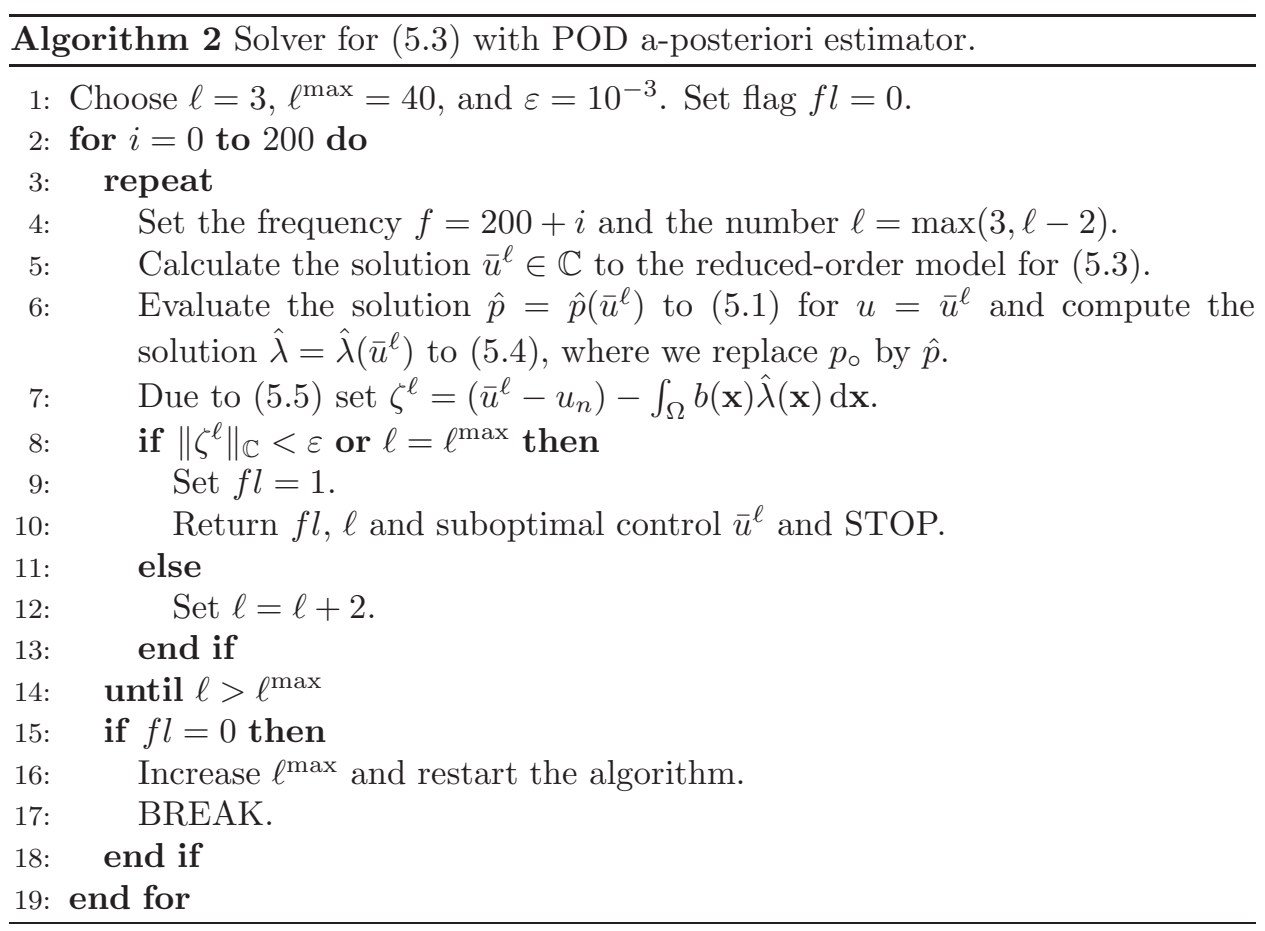

The decay of the error $\left|u_{\circ}-\bar{u}^{\ell}\right|_{\mathbb{C}}$ and the estimator $\left|\zeta^{\ell}\right|_{\mathbb{C}}$ are presented in Table 3 for fixed frequency $f=400[\mathrm{~Hz}]$. It turns out that in this example the estimate is very close to the actual error in the optimal control. For other frequencies $f$ in the frequency range from 200 to $400[\mathrm{~Hz}]$ the convergence behavior is similar.

\section{Appendix}

Proof of Lemma 2.4. Let $v \in L^{2}(\mathcal{J})$ be chosen arbitrarily. The claim is proven if we show

$$
\left\langle\mathcal{S}^{\star}\left(\Xi\left(z_{1}, z_{2}\right)-\Theta(\bar{y})\right), v\right\rangle_{L^{2}(\mathcal{J})}=\left\langle\mathcal{B}^{\star} \bar{p}, v\right\rangle_{L^{2}(\mathcal{J})} .
$$

Setting $w=\mathcal{S} v \in W(0, T)$ we infer $w(0)=0$. From (2.2), (2.6), (2.8)-(2.10) and $w(0)=0$ it follows that

$$
\begin{aligned}
& \left\langle\mathcal{S}^{\star}\left(\Xi\left(z_{1}, z_{2}\right)-\Theta(\bar{y})\right), v\right\rangle_{L^{2}(\mathcal{J})} \\
& =\left\langle\Xi\left(z_{1}, z_{2}\right)-\Theta(\bar{y}), \mathcal{S} v\right\rangle_{W(0, T)^{\prime}, W(0, T)}=\left\langle\Xi\left(z_{1}, z_{2}\right)-\Theta(\bar{y}), w\right\rangle_{W(0, T)^{\prime}, W(0, T)} \\
& =\alpha_{1}\left\langle z_{1}-\mathcal{C} \bar{y}, \mathcal{C} w\right\rangle_{W_{1}}+\alpha_{2}\left\langle z_{2}-\mathcal{D} \bar{y}(T), \mathcal{D} w(T)\right\rangle_{W_{2}} \\
& =\int_{0}^{T}-\left\langle\bar{p}_{t}(t), w(t)\right\rangle_{V^{\prime}, V}+a(\bar{p}(t), w(t)) \mathrm{d} t+\langle\bar{p}(T), w(T)\rangle_{H} \\
& =\int_{0}^{T}\left\langle w_{t}(t), \bar{p}(t)\right\rangle_{V^{\prime}, V}+a(w(t), \bar{p}(t)) \mathrm{d} t+\langle w(0), \bar{p}(0)\rangle_{H} \\
& =\int_{0}^{T}\langle(\mathcal{B} v)(t), \bar{p}(t)\rangle_{V^{\prime}, V} \mathrm{~d} t=\langle\mathcal{B} v, \bar{p}\rangle_{L^{2}\left(0, T ; V^{\prime}\right), L^{2}(0, T ; V)}=\left\langle\mathcal{B}^{\star} \bar{p}, v\right\rangle_{L^{2}(\mathcal{J})}
\end{aligned}
$$

so that (A.1) holds. 
Proof of Proposition 4.3. For almost all $t \in[0, T]$ let

$$
y^{\ell}-y(t)=y^{\ell}(t)-\mathcal{P}^{\ell} y(t)+\mathcal{P}^{\ell} y(t)-y(t)=\vartheta^{\ell}(t)+\varrho^{\ell}(t)
$$

where $\vartheta^{\ell}(t)=y^{\ell}(t)-\mathcal{P}^{\ell} y(t) \in V^{\ell}$ and $\varrho^{\ell}(t)=\mathcal{P}^{\ell} y(t)-y(t)$. From (4.6) we have

$$
\begin{aligned}
\left\|\varrho^{\ell}\right\|_{W(0, T)}^{2} & =\int_{0}^{T}\left\|y(t)-\mathcal{P}^{\ell} y(t)\right\|_{V}^{2}+\left\|y_{t}(t)-\mathcal{P}^{\ell} y_{t}(t)\right\|_{V^{\prime}}^{2} \mathrm{~d} t \\
& =\sum_{i=\ell+1}^{\infty} \lambda_{i}+\left\|y_{t}-\mathcal{P}^{\ell} y_{t}\right\|_{L^{2}\left(0, T ; V^{\prime}\right)}^{2} .
\end{aligned}
$$

Using (2.2) and (4.11) we obtain

$$
\frac{\mathrm{d}}{\mathrm{d} t}\left\langle\vartheta^{\ell}(t), \psi\right\rangle_{H}+a\left(\vartheta^{\ell}(t), \psi\right)=\left\langle y_{t}(t)-\mathcal{P}^{\ell} y_{t}(t), \psi\right\rangle_{V^{\prime}, V}
$$

for all $\psi \in V^{\ell}$ and almost all $t \in[0, T]$. From choosing $\psi=\vartheta^{\ell}(t),(2.1)$ and Young's inequality we find

$$
\begin{aligned}
\frac{1}{2} \frac{\mathrm{d}}{\mathrm{d} t}\left\|\vartheta^{\ell}(t)\right\|_{H}^{2}+\left\|\vartheta^{\ell}(t)\right\|_{V}^{2} & \leq\left\|y_{t}(t)-\mathcal{P}^{\ell} y_{t}(t)\right\|_{V^{\prime}}\left\|\vartheta^{\ell}(t)\right\|_{V} \\
& \leq \frac{1}{2}\left\|y_{t}(t)-\mathcal{P}^{\ell} y_{t}(t)\right\|_{V^{\prime}}^{2}+\frac{1}{2}\left\|\vartheta^{\ell}(t)\right\|_{V}^{2}
\end{aligned}
$$

which easily gives

$$
\frac{\mathrm{d}}{\mathrm{d} t}\left\|\vartheta^{\ell}(t)\right\|_{H}^{2}+\left\|\vartheta^{\ell}(t)\right\|_{V}^{2} \leq\left\|y_{t}(t)-\mathcal{P}^{\ell} y_{t}(t)\right\|_{V^{\prime}}^{2}
$$

for almost all $t \in[0, T]$. Integrating (A.4) over the interval $(0, T), t \in[0, T]$, and using (4.6) we arrive at

$$
\left\|\vartheta^{\ell}(t)\right\|_{H}^{2}+\int_{0}^{t}\left\|\vartheta^{\ell}(s)\right\|_{V}^{2} \mathrm{~d} s \leq\left\|\vartheta^{\ell}(0)\right\|_{H}^{2}+\left\|y_{t}-\mathcal{P}^{\ell} y_{t}\right\|_{L^{2}\left(0, T ; V^{\prime}\right)}^{2}
$$

for almost all $t \in[0, T]$. From $\vartheta^{\ell}(0)=y^{\ell}(0)-\mathcal{P}^{\ell} y(0)=y^{\ell}(0)-\mathcal{P}^{\ell} y_{0}$ and (A.5) we have

$$
\left\|\vartheta^{\ell}\right\|_{L^{2}(0, T ; V)}^{2} \leq\left\|y^{\ell}(0)-\mathcal{P}^{\ell} y_{0}\right\|_{H}^{2}+\left\|y_{t}-\mathcal{P}^{\ell} y_{t}\right\|_{L^{2}\left(0, T ; V^{\prime}\right)}^{2} .
$$

Utilizing (A.3) we find

$$
\left\langle\vartheta_{t}^{\ell}(t), \psi\right\rangle_{V^{\prime}, V}=\left\langle y_{t}(t)-\mathcal{P}^{\ell} y_{t}(t), \psi\right\rangle_{V^{\prime}, V}-a\left(\vartheta^{\ell}(t), \psi\right)
$$

for all $\psi \in V^{\ell}$ and almost all $t \in[0, T]$. Hence, from (2.1) and Cauchy-Schwarz inequality we derive

$$
\begin{aligned}
\left\|\vartheta^{\ell}\right\|_{L^{2}\left(0, T ; V^{\prime}\right)} & =\int_{0}^{T} \sup _{\|\varphi\|_{V}=1}\left\langle\vartheta_{t}^{\ell}(t), \varphi\right\rangle_{V^{\prime}, V} \mathrm{~d} t \\
& \leq \int_{0}^{T}\left(\left\|y_{t}(t)-\mathcal{P}^{\ell} y_{t}(t)\right\|_{V^{\prime}}+\left\|\vartheta^{\ell}(t)\right\|_{V}\right) \mathrm{d} t \\
& \leq \sqrt{T}\left(\left\|y_{t}-\mathcal{P}^{\ell} y_{t}\right\|_{L^{2}\left(0, T ; V^{\prime}\right)}+\left\|\vartheta^{\ell}\right\|_{L^{2}(0, T ; V)}\right) .
\end{aligned}
$$


Using (A.6) we find

$$
\begin{aligned}
\left\|\vartheta^{\ell}\right\|_{L^{2}\left(0, T ; V^{\prime}\right)}^{2} & \leq T\left(\left\|y_{t}-\mathcal{P}^{\ell} y_{t}\right\|_{L^{2}\left(0, T ; V^{\prime}\right)}+\left\|\vartheta^{\ell}\right\|_{L^{2}(0, T ; V)}\right)^{2} \\
& \leq 2 T\left(\left\|y_{t}-\mathcal{P}^{\ell} y_{t}\right\|_{L^{2}\left(0, T ; V^{\prime}\right)}^{2}+\left\|\vartheta^{\ell}\right\|_{L^{2}(0, T ; V)}^{2}\right) \\
& \leq 2 T\left(2\left\|y_{t}-\mathcal{P}^{\ell} y_{t}\right\|_{L^{2}\left(0, T ; V^{\prime}\right)}^{2}+\left\|y^{\ell}(0)-\mathcal{P}^{\ell} y_{0}\right\|_{H}^{2}\right) .
\end{aligned}
$$

Hence

$$
\left\|\vartheta^{\ell}\right\|_{L^{2}\left(0, T ; V^{\prime}\right)}^{2} \leq 4 T\left(\left\|y_{t}-\mathcal{P}^{\ell} y_{t}\right\|_{L^{2}\left(0, T ; V^{\prime}\right)}^{2}+\left\|y^{\ell}(0)-\mathcal{P}^{\ell} y_{0}\right\|_{H}^{2}\right) .
$$

Consequently, (A.2), (A.6) and (A.7) imply that

$$
\begin{aligned}
\left\|y-y^{\ell}\right\|_{W(0, T)}^{2} & \leq 2\left(\left\|\vartheta^{\ell}\right\|_{L^{2}(0, T ; V)}^{2}+\left\|\vartheta^{\ell}\right\|_{L^{2}\left(0, T ; V^{\prime}\right)}^{2}+\left\|\varrho^{\ell}\right\|_{W(0, T)}^{2}\right) \\
& =C\left(\left\|y^{\ell}(0)-\mathcal{P}^{\ell} y_{0}\right\|_{H}^{2}+\left\|y_{t}-\mathcal{P}^{\ell} y_{t}\right\|_{L^{2}\left(0, T ; V^{\prime}\right)}^{2}+\sum_{i=\ell+1}^{\infty} \lambda_{i}\right)
\end{aligned}
$$

with $C=4(2 T+1)$, so that the claim follows.

Proof of Proposition 4.6. As in the proof of Proposition 4.3 we write

$$
p^{\ell}(t)-p(t)=p^{\ell}(t)-\mathcal{P}^{\ell} p(t)+\mathcal{P}^{\ell} p(t)-p(t)=\theta^{\ell}(t)+\rho^{\ell}(t)
$$

for almost all $t \in[0, T]$, where $\theta^{\ell}(t)=p^{\ell}(t)-\mathcal{P}^{\ell} p(t) \in V^{\ell}$ and $\rho^{\ell}(t)=\mathcal{P}^{\ell} p(t)-p(t)$ hold. From (2.6) and (4.19) we obtain

$$
-\frac{\mathrm{d}}{\mathrm{d} t}\left\langle\theta^{\ell}(t), \psi\right\rangle_{H}+a\left(\theta^{\ell}(t), \psi\right)=\left\langle\mathcal{C}\left(y-y^{\ell}\right)(t), \mathcal{C} \psi\right\rangle_{W_{1}}+\left\langle p_{t}(t)-\mathcal{P}^{\ell} p_{t}(t), \psi\right\rangle_{V^{\prime}, V}
$$

for all $\psi \in V^{\ell}$ and almost all $t \in[0, T]$. Applying similar arguments as in the proof of Proposition 4.3 we arrive at

$$
\left\|\theta^{\ell}\right\|_{L^{2}(0, T ; V)}^{2} \leq\left\|\theta^{\ell}(T)\right\|_{H}^{2}+c_{1}^{2}\left\|y-y^{\ell}\right\|_{L^{2}(0, T ; H)}^{2}+\left\|p_{t}-\mathcal{P}^{\ell} p_{t}\right\|_{L^{2}\left(0, T ; V^{\prime}\right)}^{2},
$$

where $c_{1}=\sup _{\varphi \neq 0}\|\mathcal{C} \varphi\|_{W_{1}} /\|\varphi\|_{L^{2}(0, T ; H)}$. Utilizing $p(T)=\alpha_{2} \mathcal{D}^{\star}\left(z_{2}-\mathcal{D} y(T)\right) \in H$ we derive

$$
\begin{aligned}
0= & \left\langle p^{\ell}(T)-\alpha_{2} \mathcal{D}^{\star}\left(z_{2}-\mathcal{D} y^{\ell}(T)\right), p^{\ell}(T)-\mathcal{P}^{\ell} p(T)\right\rangle_{H} \\
= & \left\langle p^{\ell}(T)-\mathcal{P}^{\ell} p(T)+\mathcal{P}^{\ell} p(T)-\alpha_{2} \mathcal{D}^{\star}\left(z_{2}-\mathcal{D} y^{\ell}(T)\right), p^{\ell}(T)-\mathcal{P}^{\ell} p(T)\right\rangle_{H} \\
= & \left\|p^{\ell}(T)-\mathcal{P}^{\ell} p(T)\right\|_{H}^{2}+\left\langle\mathcal{P}^{\ell} p(T)-p(T), p^{\ell}(T)-\mathcal{P}^{\ell} p(T)\right\rangle_{H} \\
& +\left\langle\alpha_{2} \mathcal{D}^{\star} \mathcal{D}\left(y^{\ell}(T)-y(T)\right), p^{\ell}(T)-\mathcal{P}^{\ell} p(T)\right\rangle_{H}
\end{aligned}
$$

whivh gives

$$
\begin{aligned}
& \left\|p^{\ell}(T)-\mathcal{P}^{\ell} p(T)\right\|_{H}^{2} \\
& \quad=\left\langle p(T)-\mathcal{P}^{\ell} p(T)+\alpha_{2} \mathcal{D}^{\star} \mathcal{D}\left(y(T)-y^{\ell}(T)\right), p^{\ell}(T)-\mathcal{P}^{\ell} p(T)\right\rangle_{H} \\
& \quad \leq\left(\left\|p(T)-\mathcal{P}^{\ell} p(T)\right\|_{H}+\alpha_{2} c_{2}^{2}\left\|y(T)-y^{\ell}(T)\right\|_{H}\right)\left\|p^{\ell}(T)-\mathcal{P}^{\ell} p(T)\right\|_{H}
\end{aligned}
$$

with $c_{2}=\sup _{\chi \neq 0}\|\mathcal{D} \chi\|_{W_{2}} /\|\chi\|_{H}$. Hence,

$$
\left\|\theta^{\ell}(T)\right\|_{H}=\left\|p^{\ell}(T)-\mathcal{P}^{\ell} p(T)\right\|_{H} \leq\left\|p(T)-\mathcal{P}^{\ell} p(T)\right\|_{H}+\alpha_{2} c_{2}^{2}\left\|y(T)-y^{\ell}(T)\right\|_{H}
$$


Consequently, there exists a constant $C>0$ such that

$$
\begin{aligned}
\left\|\theta^{\ell}\right\|_{L^{2}(0, T ; V)}^{2} \leq & C\left(\left\|p(T)-\mathcal{P}^{\ell} p(T)\right\|_{H}^{2}+\left\|p_{t}-\mathcal{P}^{\ell} p_{t}\right\|_{L^{2}(0, T ; V)}^{2}\right) \\
& +C\left(\left\|y(T)-y^{\ell}(T)\right\|_{H}^{2}+\left\|y-y^{\ell}\right\|_{L^{2}(0, T ; H)}^{2}\right)
\end{aligned}
$$

so that (4.21) follows. By assumption, $p \in H^{1}(0, T ; V)$ holds. Analogously to (4.14) and (4.15) we find

$$
\begin{aligned}
\lim _{\ell \rightarrow \infty}\left\|p-\mathcal{P}^{\ell} p\right\|_{W(0, T)}^{2} & =\left\|p-\mathcal{P}^{\ell} p\right\|_{L^{2}(0, T ; V)}^{2}+\left\|p_{t}-\mathcal{P}^{\ell} p_{t}\right\|_{L^{2}\left(0, T ; V^{\prime}\right)}^{2} \\
& \leq C \lim _{\ell \rightarrow \infty} \int_{0}^{T} \sum_{i=\ell+1}^{\infty}\left(\left|\left\langle p(t), \psi_{i}\right\rangle_{V}\right|^{2}+\left|\left\langle p_{t}(t), \psi_{i}\right\rangle_{V}\right|^{2}\right) \mathrm{d} t=0
\end{aligned}
$$

From Proposition 4.4, Remark 4.5 and (4.21) we have $\lim _{\ell \rightarrow \infty}\left\|p-p^{\ell}\right\|_{L^{2}(0, T ; V)}=0$.

Proof of Proposition 4.8. Let $\bar{u}$ and $\bar{u}^{\ell}$ be the optimal solutions to $(\hat{\mathbf{P}})$ and $\left(\hat{\mathbf{P}}^{\ell}\right)$, respectively. From (2.7) and (4.22) we find

$$
\left\langle\sigma \bar{u}-\mathcal{B}^{\star} \bar{p}, \bar{u}^{\ell}-\bar{u}\right\rangle_{L^{2}(\mathcal{J})} \geq 0 \quad \text { and } \quad\left\langle\sigma \bar{u}^{\ell}-\mathcal{B}^{\star} \bar{p}^{\ell}, \bar{u}-\bar{u}^{\ell}\right\rangle_{L^{2}(\mathcal{J})} \geq 0 .
$$

Adding both inequalities we deduce

$$
\left\langle\sigma\left(\bar{u}-\bar{u}^{\ell}\right)+\mathcal{B}^{\star}\left(\bar{p}^{\ell}-\bar{p}\right), \bar{u}^{\ell}-\bar{u}\right\rangle_{L^{2}(\mathcal{J})} \geq 0 .
$$

Applying Lemma 2.4 and (4.20) it follows that

$$
\begin{aligned}
\sigma\left\|\bar{u}-\bar{u}^{\ell}\right\|_{L^{2}(\mathcal{J})}^{2} & \leq\left\langle\mathcal{B}^{\star}\left(\bar{p}^{\ell}-\bar{p}\right), \bar{u}^{\ell}-\bar{u}\right\rangle_{L^{2}(\mathcal{J})} \\
& =\left\langle\left(S^{\ell}\right)^{\star}\left(\Xi\left(z_{1}, z_{2}\right)-\Theta\left(\bar{y}^{\ell}\right)\right)-\mathcal{S}^{\star}\left(\Xi\left(z_{1}, z_{2}\right)-\Theta(\bar{y})\right), \bar{u}^{\ell}-\bar{u}\right\rangle_{L^{2}(\mathcal{J})} \\
& =\left\langle\left(\left(S^{\ell}\right)^{\star}-\mathcal{S}^{\star}\right) \Xi\left(z_{1}, z_{2}\right)+\mathcal{S}^{\star} \Theta(\bar{y})-\left(S^{\ell}\right)^{\star} \Theta\left(\bar{y}^{\ell}\right), \bar{u}^{\ell}-\bar{u}\right\rangle_{L^{2}(\mathcal{J})} .
\end{aligned}
$$

Recall that $\bar{y}=\hat{y}_{0}+\mathcal{S} \bar{u}$ and $\bar{y}^{\ell}=\hat{y}_{0}^{\ell}+\mathcal{S}^{\ell} \bar{u}^{\ell}$ holds. Since $\Theta$ is a linear operator, we obtain

$$
\begin{aligned}
\left\langle\mathcal{S}^{\star} \Theta(\bar{y})-\left(S^{\ell}\right)^{\star} \Theta\left(\bar{y}^{\ell}\right), \bar{u}^{\ell}-\bar{u}\right\rangle_{L^{2}(\mathcal{J})}= & \left\langle\mathcal{S}^{\star} \Theta\left(\hat{y}_{0}\right)-\left(S^{\ell}\right)^{\star} \Theta\left(\hat{y}_{0}^{\ell}\right), \bar{u}^{\ell}-\bar{u}\right\rangle_{L^{2}(\mathcal{J})} \\
& +\left\langle\mathcal{S}^{\star} \Theta(\mathcal{S} \bar{u})-\left(S^{\ell}\right)^{\star} \Theta\left(\mathcal{S}^{\ell} \bar{u}^{\ell}\right), \bar{u}^{\ell}-\bar{u}\right\rangle_{L^{2}(\mathcal{J})} .
\end{aligned}
$$

From

$$
\begin{aligned}
& \left\langle\mathcal{S}^{\star} \Theta(\mathcal{S} \bar{u})-\left(S^{\ell}\right)^{\star} \Theta\left(\mathcal{S}^{\ell} \bar{u}^{\ell}\right), \bar{u}^{\ell}-\bar{u}\right\rangle_{L^{2}(\mathcal{J})} \\
& \quad=\left\langle\mathcal{S}^{\star} \Theta(\mathcal{S} \bar{u})-\left(S^{\ell}\right)^{\star} \Theta\left(\mathcal{S}^{\ell} \bar{u}\right), \bar{u}^{\ell}-\bar{u}\right\rangle_{L^{2}(\mathcal{J})}+\left\langle\mathcal{S}_{\ell}^{\star} \Theta\left(\mathcal{S}^{\ell} \bar{u}\right)-\left(S^{\ell}\right)^{\star} \Theta\left(\mathcal{S}^{\ell} \bar{u}^{\ell}\right), \bar{u}^{\ell}-\bar{u}\right\rangle_{L^{2}(\mathcal{J})}
\end{aligned}
$$

and

$$
\begin{array}{r}
\left\langle\mathcal{S}_{\ell}^{\star} \Theta\left(\mathcal{S}^{\ell} \bar{u}\right)-\left(S^{\ell}\right)^{\star} \Theta\right. \\
\left.\left(\mathcal{S}^{\ell} \bar{u}^{\ell}\right), \bar{u}^{\ell}-\bar{u}\right\rangle_{L^{2}(\mathcal{J})}=-\left\langle\Theta\left(\mathcal{S}^{\ell}\left(\bar{u}-\bar{u}^{\ell}\right)\right),\left(S^{\ell}\right)^{\star}\left(\bar{u}-\bar{u}^{\ell}\right)\right\rangle_{L^{2}(\mathcal{J})} \\
=-\alpha_{1}\left\|\mathcal{C}\left(S^{\ell}\right)^{\star}\left(\bar{u}-\bar{u}^{\ell}\right)\right\|_{W_{1}}^{2}-\alpha_{2}\left\|\mathcal{D}\left(S^{\ell}\right)^{\star}\left(\bar{u}-\bar{u}^{\ell}\right)(T)\right\|_{W_{2}}^{2} \leq 0
\end{array}
$$


we conclude that

$$
\begin{aligned}
\sigma\left\|\bar{u}-\bar{u}^{\ell}\right\|_{L^{2}(\mathcal{J})}^{2} \leq & \left\langle\mathcal{S}^{\star}\left(\Theta(\bar{y})-\Xi\left(z_{1}, z_{2}\right)\right), \bar{u}^{\ell}-\bar{u}\right\rangle_{L^{2}(\mathcal{J})} \\
& +\left\langle\left(S^{\ell}\right)^{\star}\left(\Xi\left(z_{1}, z_{2}\right)-\Theta\left(\hat{y}_{0}^{\ell}+\mathcal{S}^{\ell} \bar{u}\right)\right), \bar{u}^{\ell}-\bar{u}\right\rangle_{L^{2}(\mathcal{J})} \\
= & \left\langle\mathcal{B}^{\star}\left(\bar{p}-\hat{p}^{\ell}\right), \bar{u}^{\ell}-\bar{u}\right\rangle_{L^{2}(\mathcal{J})} \\
\leq & \|\mathcal{B}\|_{L\left(L^{2}(\mathcal{J}), L^{2}(0, T ; V)\right)}\left\|\bar{p}-\hat{p}^{\ell}\right\|_{L^{2}(0, T ; V)}\left\|\bar{u}-\bar{u}^{\ell}\right\|_{L^{2}(\mathcal{J})},
\end{aligned}
$$

where $\hat{p}^{\ell}$ solves (4.24) and $\hat{y}^{\ell}$ is the solution to (4.25). Thus, (A.8) implies that

$$
\left\|\bar{u}-\bar{u}^{\ell}\right\|_{L^{2}(\mathcal{J})} \leq c\left\|\bar{p}-\hat{p}^{\ell}\right\|_{L^{2}(0, T ; V)} .
$$

with the constant $c=\|\mathcal{B}\|_{L\left(L^{2}(\mathcal{J}), L^{2}(0, T ; V)\right)} / \sigma$.

Acknowledgement. S. V. would like to thank Dr. Achim Hepberger of ACC, Acoustic Competence Center G.m.B.H., Graz, for providing measurements and data for Run 2 in Section 5.

\section{REFERENCES}

[1] N. Arada, E. Casas, and F. Tröltzsch. Error estimates for the numerical approximation of a semilinear elliptic control problem. Computational Optimization and Applications, 23:201$229,2002$.

[2] J.A. Atwell, J.T. Borggaard, and B.B. King. Reduced order controllers for Burgers' equation with a nonlinear observer. Int. J. Appl. Math. Comput. Sci., 11:1311-1330, 2001.

[3] E. Arian, M. Fahl, and E.W. Sachs. Trust-region proper orthogonal decomposition for flow control. Technical Report 2000-25, ICASE, 2000.

[4] P. Benner and E.S. Quintana-Ortí. Model reduction based on spectral projection methods. In Reduction of Large-Scale Systems, P. Benner, V. Mehrmann, D. C. Sorensen (eds.), Lecture Notes in Computational Science and Engineering, 45, 5-48, 2005.

[5] E. Casas and F. Tröltzsch. Error estimates for the finite-element approximation of a semilinear elliptic control problem. Control and Cybernetics 31:695-712, 2002.

[6] R. Dautray and J.-L. Lions. Mathematical Analysis and Numerical Methods for Science and Technology. Volume 5: Evolution Problems I. Springer-Verlag, Berlin, 1992.

[7] F. Diwoky and S. Volkwein. Nonlinear boundary control for the heat equation utilizing proper orthogonal decomposition. International Series of Numerical Mathematics, 138:73-87, 2001.

[8] T. Henri and M. Yvon. Convergence estimates of POD Galerkin methods for parabolic problems. Technical Report No. 02-48, Institute of Mathematical Research of Rennes, 2002.

[9] A. Hepberger. Mathematical methods for the prediction of the interior car noise in the middle frequency range. PhD tesis, TU Graz, Institute for Mathematics, Austria, 2002.

[10] A. Hepberger, S. Volkwein, F. Diwoky, and H.-H. Priebsch. Impedance identification out of pressure datas with a hybrid measurement-simulation methodology up to $1 \mathrm{kHz}$. In Proceedings of International Conference on Noise and Vibration Engineering, Leuven, Belgium, 2006.

[11] M. Hintermüller, K. Ito, and K. Kunisch. The primal-dual active set strategy as a semi-smooth Newton method. SIAM J. Optimization, 13:865-888, 2003.

[12] M. Hinze and S. Volkwein. Error estimates for abstract linear-quadratic optimal control problems using proper orthogonal decomposition. Computational Optimization and Applications, to appear.

[13] P. Holmes, J.L. Lumley, and G. Berkooz. Turbulence, Coherent Structures, Dynamical Systems and Symmetry. Cambridge Monographs on Mechanics, Cambridge University Press, 1996.

[14] T. Kato. Perturbation Theory for Linear Operators. Springer-Verlag, Berlin, 1980.

[15] K. Kunisch and S. Volkwein. Proper orthogonal decomposition for optimality systems. ESAIM: Mathemematical Modelling and Numerical Analysis, to appear.

[16] K. Kunisch and S. Volkwein. Galerkin proper orthogonal decomposition methods for parabolic problems. Numerische Mathematik, 90:117-148, 2001. 
[17] K. Kunisch and S. Volkwein. Galerkin proper orthogonal decomposition methods for a general equation in fluid dynamics. SIAM J. Numer. Anal., 40:492-515, 2002.

[18] S. Lall, J.E. Marsden, and S. Glavaski. A subspace approach to balanced truncation for model reduction of nonlinear control systems. Int. J. Robust Nonlinear Control, 12:519-535, 2002.

[19] J.L. Lions. Optimal Control of Systems Governed by Partial Differential Equations. Springer, Berlin, 1971.

[20] H.V. Ly and H.T. Tran. Modeling and control of physical processes using proper orthogonal decomposition. Mathematical and Computer Modeling, 33:223-236, 2001.

[21] K. Malanowski, C. Büskens, H. Maurer. Convergence of approximations to nonlinear control problems. In: Mathematical Programming with Data Perturbation, ed.: A. V. Fiacco; Marcel Dekker, Inc., New York (1997), 253-284.

[22] J. Nocedal and S.J. Wright. Numerical Optimization. Springer Series in Operation Research, Second Edition, 2006.

[23] S.S. Ravindran. Adaptive reduced order controllers for a thermal flow system using proper orthogonal decomposition. SIAM J. Sci. Comput., 28:1924-1942, 2002.

[24] M. Read and B. Simon. Methods of Modern Mathematical Physics I: Functional Analysis Academic Press, Boston, 1980.

[25] C.W. Rowley. Model reduction for fluids, using balanced proper orthogonal decomposition. Int. J. on Bifurcation and Chaos, 15:997-1013, 2005.

[26] L. Sirovich. Turbulence and the dynamics of coherent structures, parts I-III. Quart. Appl. Math., XLV:561-590, 1987.

[27] S. Volkwein. Model Reduction using Proper Orthogonal Decomposition. Lecture Notes, Institute of Mathematics and Scientific Computing, University of Graz. see http://www.uni-graz.at/imawww/volkwein/POD.pdf

[28] S. Volkwein and A. Hepberger. Impedance identification by POD model reduction techniques. Submitted, 2007.

[29] K. Willcox and J. Peraire. Balanced model reduction via the proper orthogonal decomposition. American Institute of Aeronautics and Astronautics (AIAA), 2323-2330, 2002.

F. Tröltzsch, Institute of Mathematics, Faculty II - Mathematics and Natural Sciences, Berlin University of Technology, Strasse des 17. Juni 136, D-10623 Berlin, GERMANY

E-mail address: troeltz@math.tu-berlin.de

S. Volkwein, Institute for Mathematics and Scientific Computing, University of Graz, Heinrichstrasse 36, 8010 Graz, Austria

E-mail address: stefan.volkwein@uni-graz.at 